\title{
On the parallel between normality and extremal disconnectedness ${ }^{\text {th }}$
}

\author{
Javier Gutiérrez García ${ }^{\mathrm{a}}$ Jorge Picado ${ }^{\mathrm{b}, *}$ \\ ${ }^{a}$ Department of Mathematics, University of the Basque Country UPV/EHU, Apdo. 644, 48080 Bilbao, Spain \\ ${ }^{b}$ CMUC, Department of Mathematics, University of Coimbra, 3001-501 Coimbra, Portugal
}

\begin{abstract}
Several familiar results about normal and extremally disconnected (classical or pointfree) spaces shape the idea that the two notions are somehow dual to each other and can therefore be studied in parallel. This paper investigates the source of this 'duality' and shows that each pair of parallel results can be framed by the 'same' proof. The key tools for this purpose are relative notions of normality, extremal disconnectedness, semicontinuity and continuity (with respect to a fixed class of complemented sublocales of the given locale) that bring and extend to locale theory a variety of well-known classical variants of normality and upper and lower semicontinuities in a illuminating unified manner. This approach allows us to unify under a single localic proof all classical insertion, as well as their corresponding extension results.
\end{abstract}

Keywords: Locale theory, sublocale lattice, localic map, frame, normal frame, extremally disconnected frame, completely separated sublocales, Katětov relation, continuous real function, normal, regular and zero continuous real functions, upper and lower semicontinuous real functions, continuous extension.

2010 MSC: 06D22, 06D30, 26A15, 54C30, 54D15, 54G05.

\section{Introduction}

In Real Analysis, in spaces like $\mathbb{R}$ or $\mathbb{R}^{2}$, we are used to a great abundance of continuous maps with real values. But there are non-trivial spaces that do not admit continuous real-valued functions other than the constant ones. The abundance of real continuous functions in a space (or locale) can be assessed by the existence of functions that indeed separate all subsets (or sublocales) that can possibly be separated, and the (separation) lemma of Urysohn characterizes those spaces and locales ("with plenty of continuous real functions" [9]): they are precisely the

\footnotetext{
${ }^{2}$ Research supported by the Ministry of Economy and Competitiveness of Spain (under grant MTM2012-37894C02-02), the UPV/EHU (under grants UFI11/52 and GIU12/39) and the Centre for Mathematics of the University of Coimbra (funded by the European Regional Development Fund through the program COMPETE and by the Portuguese Government through the Fundação para a Ciência e a Tecnologia, under the project PEst-C/MAT/UI0324/2011).

${ }^{*}$ Corresponding author

Email addresses: javier.gutierrezgarcia@ehu.es (Javier Gutiérrez García), picado@mat .uc.pt (Jorge Picado)

URL: www. ehu.es/javiergutierrezgarcia (Javier Gutiérrez García), www . mat .uc .pt/ picado (Jorge Picado)
} 
normal ones. Extremally disconnected (De Morgan) spaces or locales are also very important (see [27]).

As observed by T. Kubiak in [33], several pairs of results in classical topology like those in Table 1 characterizing the concepts of normality and extremal disconnectedness show a "remarkable duality" (in the words of [33]) between the two concepts: each pair is identical in structure but prove facts about normal spaces on one side of the pair and about extremally disconnected spaces on the other. The origin of this observation goes back to [30] and it also appears in [31] and [29, p. 301] (consult [25] for more examples of results of this kind in the setting of quasiuniform spaces). Nevertheless the known proofs of the results in each pair are quite different in nature (and the same happens with the proofs of the results in [25]), requiring even in some cases different tools and constructions.

\begin{tabular}{|c|c|c|}
\hline Space $X$ & NORMAL & EXtREMALly DisconNECTED \\
\hline $\begin{array}{l}\text { Urysohn's } \\
\text { separation type } \\
\text { lemma }\end{array}$ & $\begin{array}{l}\text { Every two disjoint CLOSED } \\
\text { subsets of } X \text { are completely } \\
\text { separated (Urysohn [47]). }\end{array}$ & $\begin{array}{l}\text { Every two disjoint open subsets } \\
\text { of } X \text { are completely separated } \\
\text { (Gillman and Jerison [12]). }\end{array}$ \\
\hline $\begin{array}{l}\text { Tietze's extension } \\
\text { type theorem }\end{array}$ & $\begin{array}{l}\text { Each closed subset of } X \text { is } \\
C^{*} \text {-embedded (Tietze [45]). }\end{array}$ & $\begin{array}{l}\text { Each open subset of } X \text { is } \\
C^{*} \text {-embedded (Gillman and } \\
\text { Jerison [12]). }\end{array}$ \\
\hline $\begin{array}{l}\text { Katětov-Tong } \\
\text { insertion type } \\
\text { theorem }\end{array}$ & $\begin{array}{l}\text { For every UPPER semicontinuous } \\
\text { real function } f \text { and LOWER semi- } \\
\text { continuous real function } g \text { satis- } \\
\text { fying } f \leq g \text {, there exists a con- } \\
\text { tinuous real function } h \text { such that } \\
f \leq h \leq g \text { (Katětov [28], Tong } \\
\text { [46]). }\end{array}$ & $\begin{array}{l}\text { For every LOWER semicontinuous } \\
\text { real function } f \text { and UPPER semi- } \\
\text { continuous real function } g \text { satis- } \\
\text { fying } f \leq g \text {, there exists a con- } \\
\text { tinuous real function } h \text { such that } \\
f \leq h \leq g \text { (Stone [44], Lane } \\
[34]) .\end{array}$ \\
\hline $\begin{array}{l}\text { Hausdorff mapping } \\
\text { invariance type } \\
\text { theorem }\end{array}$ & $\begin{array}{l}\text { The image of } X \text { under any } \\
\text { CLOSED continuous map is } \\
\text { NORMAL (Hausdorff [23]). }\end{array}$ & $\begin{array}{l}\text { The image of } X \text { under any OPEN } \\
\text { continuous map is EXTREMALLY } \\
\text { DISCONNECTED. }\end{array}$ \\
\hline
\end{tabular}

Table 1: Characterizations of normal and extremally disconnected spaces

Our recent work in the more general localic setting (see e.g. [20, 15, 17]) reveals a similar picture, summarized in Table 2.

This shapes the idea that the two notions are somehow dual to each other and therefore may be studied in parallel; hopefully, one may even find 'dual' proofs for each pair of results. It is the aim of this paper to examine this parallel. In particular, we address the following questions:

(1) What is the source of this duality?

(2) The proofs of the results in each pair are very different in nature. Can one unify them under the same result with a single proof?

(3) There is a great variety of classical insertion type results (for several variants of normality). Can one unify them under a single general result? 


\begin{tabular}{|c|c|c|}
\hline Locale $L$ & Normal & ExtREMAlly DisconNected \\
\hline $\begin{array}{l}\text { Urysohn's } \\
\text { separation type } \\
\text { lemma }\end{array}$ & $\begin{array}{l}\text { Every two disjoint CLOSED } \\
\text { sublocales of } L \text { are completely } \\
\text { separated. }\end{array}$ & $\begin{array}{l}\text { Every two disjoint oPEN } \\
\text { sublocales of } L \text { are completely } \\
\text { separated. }\end{array}$ \\
\hline $\begin{array}{l}\text { Tietze's extension } \\
\text { type theorem }\end{array}$ & $\begin{array}{l}\text { Each cLosed sublocale of } L \text { is } \\
C^{*} \text {-embedded. }\end{array}$ & $\begin{array}{l}\text { Each open sublocale of } L \text { is } \\
C^{*} \text {-embedded. }\end{array}$ \\
\hline $\begin{array}{l}\text { Katětov-Tong } \\
\text { insertion type } \\
\text { theorem }\end{array}$ & $\begin{array}{l}\text { For every UPPER semicontinuous } \\
\text { real function } f \text { and LOWER semi- } \\
\text { continuous real function } g \text { satis- } \\
\text { fying } f \leq g \text {, there exists a con- } \\
\text { tinuous real function } h \text { such that } \\
f \leq h \leq g \text {. }\end{array}$ & $\begin{array}{l}\text { For every LOWER semicontinuous } \\
\text { real function } f \text { and UPPER semi- } \\
\text { continuous real function } g \text { satis- } \\
\text { fying } f \leq g \text {, there exists a con- } \\
\text { tinuous real function } h \text { such that } \\
f \leq h \leq g \text {. }\end{array}$ \\
\hline
\end{tabular}

Table 2: Characterizations of normal and extremally disconnected locales

(4) Can one complete Table 2 with a pointfree extension of Hausdorff mapping invariance type theorems?

The main idea will be to fix a class $\mathscr{A}$ of complemented sublocales of a locale $L$. Depending on the parameter $\mathscr{A}$, we introduce and study dual relative notions of normality and extremal disconnectedness (respectively $\mathscr{A}$-normality and $\mathscr{A}$-disconnectedness) and notions of $\mathscr{A}$-continuous and lower and upper $\mathscr{A}$-semicontinuous real functions on $L$. Taking for $\mathscr{A}$ the standard closed sublocales, one obtains the usual notions. By varying the choice of $\mathscr{A}$, we reach a wide array of examples.

Since every complemented sublocale of a space is a subspace [24], in the case that the locale $L$ is the lattice $O X$ of open subsets of some space $X$, these notions can be completely formulated in terms of the space $X$, with no reference to sublocales, and provide a unification of the most relevant classical notions in the literature $[8,10,28,34,35,36,37,41,43,46]$ (some of them are here introduced and studied for the first time in the pointfree setting).

Our results characterize $\mathscr{A}$-normal locales and generalize all characterizations in Table 2. They hold for any class $\mathscr{A}$ that induces a Katětov relation on the lattice of all sublocales. Then the dual results for $\mathscr{A}$-disconnectedness correspond to the results for the class $\mathscr{A}^{\mathrm{c}}$ of complements of elements of $\mathscr{A}$ and are therefore obtained with no extra cost. Again, this approach allows to extend and unify the most relevant classical insertion results [8, 28, 46, 35, 44].

By relativizing the notion of an extension of a real function on a sublocale to the whole locale, we obtain a relative form of Tietze's extension theorem and the corresponding dual result. In addition we also prove a relative version for the preservation of normality under localic maps that extends the Hausdorff mapping invariance type theorems of Table 1 to the pointfree setting, thus completing Table 2.

There is one important aspect of insertion and normality which is not considered in this paper, namely strict insertion $[16,21]$ and its connection with variants of perfect normality. This will be treated in a separate paper [18]. 


\section{Background and notation}

We take the localic approach to topology. If $X$ is a topological space, the partially ordered set $O X$ of open subsets of $X$ is a complete lattice, in which the infinite distributive law

$$
U \wedge \bigvee \mathcal{S}=\bigvee\{U \wedge S \mid S \in \mathcal{S}\}
$$

holds for all open subsets $U$ and collections of open subsets $\mathcal{S}$ in $X$. We recall that a frame is an abstract complete lattice with these properties; like inverse image along a continuous mapping, a frame homomorphism is taken to preserve arbitrary joins (including the bottom element 0 of the lattice) and finite meets (including the top element 1 of the lattice). We write Frm for the category of frames and frame homomorphisms.

The above representation is contravariant: continuous maps $f: X \rightarrow Y$ are represented by frame homomorphisms $h: O Y \rightarrow O X$. This is easily mended, in order to keep the geometric motivation, by considering, instead of Frm, its opposite category Loc of locales and localic maps. In Loc we have "generalized continuous maps" $f: L \rightarrow M$ that can be regarded as frame homomorphisms $h: L \leftarrow M$. In most of the paper we keep the algebraic (frame) approach. When dealing with images and preimages in Section 9, however, we have found the localic covariant approach (see [39] for more information about it) more useful, enabling us to write the proof of the main result in a very short and transparent way.

Any frame $L$ is in particular a complete Heyting algebra (with Heyting implication $\rightarrow$ ) so there are the pseudocomplements

$$
a^{*}=a \rightarrow 0=\bigvee\{b \in L \mid a \wedge b=0\}
$$

satisfying $a \wedge a^{*}=0$. Whenever $a^{*}$ is a complement of $a$ (that is, $a^{*} \vee a=1$ ) we shall denote it by $a^{c}$. An element $a$ is regular if $a^{* *}=a$ (equivalently, if $a=b^{*}$ for some $b$ ).

For basic facts about pointfree topology and lattice theory we refer to [27] and [40]. Below we recall some details of specific relevance to this paper.

The sublocale lattice

A sublocale set (briefly, a sublocale) $S$ of a frame $L$ is a subset $S \subseteq L$ such that

(S1) for every $A \subseteq S, \wedge A$ is in $S$, and

(S2) for every $s \in S$ and every $x \in L, x \rightarrow s$ is in $S$.

In the lattice of sublocales of $L$ the least element is $\{1\}$ and the largest one is $L$. The meets coincide with intersections and the joins are given by the formula $\bigvee_{i \in I} S_{i}=\left\{\wedge A \mid A \subseteq \bigcup_{i \in I} S_{i}\right\}$.

This is a co-frame (i.e., its dual lattice is a frame). In the sequel, we make it into a frame $\mathcal{S}(L)$ by considering the dual ordering $S_{1} \leq S_{2}$ iff $S_{2} \subseteq S_{1}$. Thus, given $\left\{S_{i} \in \mathcal{S}(L): i \in I\right\}$, we have $\bigvee_{i \in I} S_{i}=\bigcap_{i \in I} S_{i}$ and $\bigwedge_{i \in I} S_{i}=\left\{\bigwedge A \mid A \subseteq \bigcup_{i \in I} S_{i}\right\}$. Also, $\{1\}$ is the top and $L$ is the bottom in $\mathcal{S}(L)$ that we simply denote by 1 and 0 , respectively.

For any $a \in L$, the sets $\mathfrak{c}(a)=\uparrow a$ and $\mathfrak{v}(a)=\{a \rightarrow b: b \in L\}$ are the closed and open sublocales of $L$, respectively. They are complements of each other in $\mathcal{S}(L)$. Furthermore, the map $a \mapsto \mathfrak{c}(a)$ is a frame embedding $L \hookrightarrow \mathcal{S}(L)$ providing an isomorphism $c$ between $L$ and the subframe $\mathfrak{c}(L)$ of $\mathcal{S}(L)$ consisting of all closed sublocales. On the other hand, denoting by $\mathfrak{v}(L)$ the sublattice of $\mathcal{S}(L)$ formed by all $\mathfrak{v}(a)$ (contrarily to $\mathfrak{c}(L)$, this is not a subframe of $\mathcal{S}(L)$ ), the correspondence $a \mapsto \mathfrak{v}(a)$ establishes a dual lattice embedding $L \rightarrow \mathfrak{v}(L)$. 
Given a sublocale $S$ of $L$, its closure and interior are defined, respectively, by

$$
\bar{S}=\bigvee\{\mathfrak{c}(a): \mathfrak{c}(a) \leq S\}=\mathfrak{c}(\bigwedge S) \quad \text { and } \quad S^{\circ}=\bigwedge\{\mathfrak{v}(a): S \leq \mathfrak{v}(a)\}
$$

They have the following properties:

Proposition 1.1. Let $S, T \in \mathcal{S}(L), a \in L$ and $A \subseteq L$. Then:

(1) $\overline{1}=1, \bar{S} \leq S, \overline{\bar{S}}=\bar{S}$, and $\overline{S \wedge T}=\bar{S} \wedge \bar{T}$,

(2) $0^{\circ}=0, S^{\circ} \geq S, S^{\circ \circ}=S^{\circ}$, and $(S \vee T)^{\circ}=S^{\circ} \vee T^{\circ}$,

(3) $S^{\circ}=\left(\overline{S^{*}}\right)^{\mathrm{c}}=\mathfrak{p}\left(\bigwedge S^{*}\right)$,

(4) $\mathfrak{c}(a)^{\circ}=\mathfrak{v}\left(a^{*}\right)$,

(5) $\overline{\mathfrak{p}(a)}=\mathfrak{c}\left(a^{*}\right)$.

A sublocale is said to be clopen if it is both closed and open. Clearly, $S$ is clopen iff $S=$ $\mathfrak{c}(a)=\mathfrak{v}\left(a^{\mathrm{c}}\right)$ for some complemented $a$ in $L$.

A $G_{\delta}$-sublocale is a countable join of open sublocales $\bigvee_{n \in \mathbb{N}} \mathfrak{D}\left(a_{n}\right)$ and an $F_{\sigma}$-sublocale is a countable meet of closed sublocales $\bigwedge_{n \in \mathbb{N}} \mathrm{c}\left(a_{n}\right)$.

\section{Real-valued functions}

The frame of reals $[3]$ is the frame $\mathfrak{L}(\mathbb{R})$ generated by all pairs $(p, q) \in \mathbb{Q} \times \mathbb{Q}$ satisfying the relations

(R1) $(p, q) \wedge(r, s)=(p \vee r, q \wedge s)$,

(R2) $(p, q) \vee(r, s)=(p, s)$ whenever $p \leq r<q \leq s$,

(R3) $(p, q)=\bigvee\{(r, s): p<r<s<q\}$,

(R4) $\bigvee_{p, q \in \mathbb{Q}}(p, q)=1$.

We use the following notation: $(p,-)=\bigvee_{q \in \mathbb{Q}}(p, q)$ and $(-, q)=\bigvee_{p \in \mathbb{Q}}(p, q)$; note that $(p,-) \wedge$ $(-, q)=(p, q)$.

Equivalently, $\mathfrak{Q}(\mathbb{R})$ may be defined as the frame with generators of the form $(r,-)$ and $(-, r)$, $r \in \mathbb{Q}$, subject to relations

(r1) $(r,-) \wedge(-, s)=0$ whenever $r \geq s$,

(r2) $(r,-) \vee(-, s)=1$ whenever $r<s$,

(r3) $(r,-)=\bigvee_{s>r}(s,-)$, for every $r \in \mathbb{Q}$,

(r4) $(-, r)=\bigvee_{s<r}(-, s)$, for every $r \in \mathbb{Q}$,

(r5) $\bigvee_{r \in \mathbb{Q}}(r,-)=1$,

(r6) $\bigvee_{r \in \mathbb{Q}}(-, r)=1$.

With $(p, q)=(p,-) \wedge(-, q)$ one goes back to $(\mathrm{R} 1)-(\mathrm{R} 4)$.

Recall from [17] that a (general) real-valued function on $L$ is a frame homomorphism $\mathfrak{Q}(\mathbb{R}) \rightarrow$ $\mathcal{S}(L)$. The class of all real functions on $L$ is denoted by $\mathrm{F}(L)$. It is partially ordered by

$$
\begin{array}{rlrl}
f \leq g & \Leftrightarrow \quad f(p,-) \leq g(p,-) & & \text { for every } p \in \mathbb{Q} \\
& \Leftrightarrow g(-, q) \leq f(-, q) & \text { for every } q \in \mathbb{Q} .
\end{array}
$$


For each $r \in \mathbb{Q}$, we denote by $\boldsymbol{r}$ the constant real-valued function defined for each $p, q \in \mathbb{Q}$ by

$$
\boldsymbol{r}(p,-)=\left\{\begin{array}{ll}
1 & \text { if } p<r \\
0 & \text { if } p \geq r
\end{array} \text { and } \quad \boldsymbol{r}(-, q)= \begin{cases}0 & \text { if } q \leq r \\
1 & \text { if } q>r .\end{cases}\right.
$$

Furthermore, let $S$ be a complemented sublocale of $L$. Then $\chi_{S}$ defined for each $p, q \in \mathbb{Q}$ by

$$
\chi_{S}(p,-)=\left\{\begin{array}{ll}
1 & \text { if } p<0 \\
S^{c} & \text { if } 0 \leq p<1 \\
0 & \text { if } p \geq 1
\end{array} \quad \text { and } \quad \chi_{S}(-, q)= \begin{cases}1 & \text { if } q>1 \\
S & \text { if } 0<q \leq 1 \\
0 & \text { if } q \leq 0\end{cases}\right.
$$

belongs to $\mathrm{F}(L)$ and it is called the characteristic function of $S$.

Scales in $\mathcal{S}(L)$

In order to define an $f \in \mathrm{F}(L)$ it suffices to consider a map from the set of generators $\{(r,-),(-, r) \mid r \in \mathbb{Q}\}$ of $\mathfrak{Q}(\mathbb{R})$ to $\mathcal{S}(L)$ that turns the defining relations (r1)-(r6) into identities in $\mathcal{S}(L)$. This can be easily done with scales. A family $\left(S_{p} \mid p \in \mathbb{Q}\right)$ of sublocales of $L$ is a scale if

(S1) $S_{p} \vee S_{q}{ }^{*}=1$ whenever $p<q$, and

(S2) $\bigvee_{p \in \mathbb{Q}} S_{p}=1=\bigvee_{p \in \mathbb{Q}} S_{p}{ }^{*}$.

As a basic fact in this context, any scale $\left(S_{r} \mid r \in \mathbb{Q}\right)$ determines an $f \in \mathrm{F}(L)$ by the formulas

$$
f(p,-)=\bigvee_{r>p} S_{r} \text { and } f(-, q)=\bigvee_{r<q} S_{r}^{*},
$$

for every $p, q \in \mathbb{Q}$.

Remarks 1.2. (1) By condition (S1) a scale is necessarily an antitone family. On the other hand, if a family $C=\left(S_{p} \mid p \in \mathbb{Q}\right)$ of sublocales of $L$ is antitone and for each $p<q$ in $\mathbb{Q}$ there exists a complemented sublocale $S_{p, q}$ such that $S_{q} \leq S_{p, q} \leq S_{p}$, then $C$ satisfies (S1). Indeed, $S_{p} \vee S_{q}{ }^{*} \geq S_{p, q} \vee S_{p, q}{ }^{\mathrm{C}}=1$ whenever $p<q$. In particular, if $C$ consists of complemented sublocales, then $C$ satisfies ( $\mathrm{S} 1)$ if and only if it is antitone.

(2) Given a real function $f \in \mathrm{F}(L)$, the families

$$
(f(r,-) \mid r \in \mathbb{Q}) \quad \text { and } \quad\left(f(-, r)^{*} \mid r \in \mathbb{Q}\right)
$$

are clearly scales. Moreover, they both generate the real function $f$.

Lemma 1.3. Let $\left(S_{r} \mid r \in \mathbb{Q}\right)$ and $\left(T_{r} \mid r \in \mathbb{Q}\right)$ be scales generating real functions $f$ and $g$, respectively. Then:

$$
f \leq g \quad \Leftrightarrow \quad S_{q} \leq T_{p} \text { for every } p<q .
$$

Proof. Let $p<q$ in $\mathbb{Q}$ and take $r \in \mathbb{Q}$ with $p<r<q$. Then $S_{q} \leq f(r,-) \leq g(r,-) \leq T_{p}$. Conversely, for each $p \in \mathbb{Q}$,

$$
f(p,-)=\bigvee_{r>p} \bigvee_{s>r} S_{s} \leq \bigvee_{r>p} T_{r}=g(p,-)
$$




\section{Stone algebras}

A Stone algebra is a distributive pseudocomplemented algebra ( $p$-algebra) $A$ that satisfies the Stone identity $a^{*} \vee a^{* *}=1$ for every $a \in A$. It is well known that the first De Morgan law $(a \vee b)^{*}=a^{*} \wedge b^{*}$ holds in any $p$-algebra while the second De Morgan law $(a \wedge b)^{*}=a^{*} \vee b^{*}$ holds only for Stone algebras:

Proposition 1.4. In any distributive p-algebra $A$ we have:

(1) $a \wedge b=0 \Leftrightarrow a^{* *} \wedge b^{* *}=0$.

(2) $(a \vee b)^{*}=a^{*} \wedge b^{*}$ for every $a, b \in A$.

(3) $(a \wedge b)^{* *}=a^{* *} \wedge b^{* *}$ for every $a, b \in A$.

(4) If $A$ is a Stone algebra then $(a \vee b)^{* *}=a^{* *} \vee b^{* *}$ for every $a, b \in A$.

(5) If $A$ is a Stone algebra then $(a \wedge b)^{*}=a^{*} \vee b^{*}$ for every $a, b \in A$.

Proof. (1) $a \wedge b=0 \Leftrightarrow a \leq b^{*} \Rightarrow a^{* *} \leq b^{* * *}=b^{*} \Rightarrow a^{* *} \wedge b^{* *} \leq b^{*} \wedge b^{* *}=0$. The converse is obvious.

(2) is straightforward.

(3) $(a \wedge b)^{* *} \leq a^{* *} \wedge b^{* *}$ is obvious. Conversely, if $x \wedge(a \wedge b)=0$ then, applying (1), we get $x \wedge a^{* *} \wedge b^{* *}=0$. For $x=(a \wedge b)^{*}$ this means that $a^{* *} \wedge b^{* *} \leq(a \wedge b)^{* *}$.

(4) $(a \vee b)^{* *} \geq a^{* *} \vee b^{* *}$ is always true. It remains to show that $x \wedge(a \vee b)^{*}=0$ implies $x \leq a^{* *} \vee b^{* *}$. Let $x \wedge a^{*} \wedge b^{*}=0$. Then $x \wedge a^{*} \leq b^{* *}$. Thus $x=x \wedge 1=x \wedge\left(a^{*} \vee a^{* *}\right)=\left(x \wedge a^{*}\right) \vee\left(x \wedge a^{* *}\right) \leq b^{* *} \vee a^{* *}$. (5) Using (2), (3) and (4) we may write $(a \wedge b)^{*}=(a \wedge b)^{* * *}=\left(a^{* *} \wedge b^{* *}\right)^{*}=\left(a^{*} \vee b^{*}\right)^{* *}=$ $a^{* * *} \vee b^{* * *}=a^{*} \vee b^{*}$.

\section{Normality versus extremal disconnectedness}

Recall that a frame $L$ is normal if

$$
a \vee b=1 \Longrightarrow \exists u, v \in L: u \wedge v=0 \text { and } a \vee u=1=b \vee v
$$

Proposition 2.1. (Cf. [11, Prop. 3.5]) The following are equivalent for a frame L:

(1) L is normal.

(2) If $\mathfrak{c}(a) \vee \mathfrak{c}(b)=1$, then there exist $u, v \in L$ such that $\mathfrak{c}(u) \wedge \mathfrak{c}(v)=0$ and $\mathfrak{c}(a) \vee \mathfrak{c}(u)=1=$ $c(b) \vee c(v)$

(3) If $\mathfrak{v}(a) \wedge \mathfrak{v}(b)=0$, then there exist $u, v \in L$ such that $\mathfrak{v}(u) \vee \mathfrak{v}(v)=1$ and $\mathfrak{v}(a) \wedge \mathfrak{v}(u)=0=$ $\mathfrak{D}(b) \wedge \mathfrak{D}(v)$.

(4) The frame $\uparrow a$ is normal for every $a \in L$.

Proof. (1) $\Leftrightarrow(2) \Leftrightarrow(3)$ and (4) $\Rightarrow(1)$ are trivial

$(1) \Rightarrow(4)$ : Let $x, y \in \uparrow a$ satisfying $x \vee y=1$. By hypothesis, there exist $u, v \in L$ such that $u \wedge v=0$ and $x \vee u=1=y \vee v$. It is then obvious that $\bar{u}=u \vee a \in \uparrow a$ and $\bar{v}=v \vee a \in \uparrow a$ satisfy $\bar{u} \wedge \bar{v}=a$ and $x \vee \bar{u}=1=y \vee \bar{v}$. 
Remark 2.2. In any normal frame the rather below relation

$$
a<b \equiv a^{*} \vee b=1
$$

is interpolative. Indeed, if $a^{*} \vee b=1$ then there exist $u, v \in L$ satisfying $u \wedge v=0$ and $a^{*} \vee u=$ $1=b \vee v$, and thus $a<u<b$ (since $u^{*} \vee b \geq v \vee b=1$ ).

Note that when $<$ interpolates, it coincides with the relation $\ll$ where $a \ll b$ expresses the familiar relation that $a$ is really inside, or completely below, $b$ (Johnstone [27]).

On the other hand, a frame $L$ is said to be extremally disconnected (also De Morgan) if it is a Stone algebra, that is, $a^{*} \vee a^{* *}=1$ for every $a \in L$ (in other words, every regular element of $L$ is complemented) $[27,38]$. We observe that this notion is in accordance with the corresponding classical one for a topological space $X$ : a space $X$ is extremally disconnected iff the frame $O X$ is extremally disconnected.

Proposition 2.3. The following are equivalent for a frame $L$ :

(1) L is extremally disconnected.

(2) The second De Morgan law holds in L.

(3) If $a \wedge b=0$, then there exist $u, v \in L$ such that $u \vee v=1$ and $a \wedge u=0=b \wedge v$.

(4) If $\mathfrak{c}(a) \wedge \mathfrak{c}(b)=0$, then there exist $u, v \in L$ such that $\mathfrak{c}(u) \vee \mathfrak{c}(v)=1$ and $\mathfrak{c}(a) \wedge \mathfrak{c}(u)=0=$ $\mathfrak{c}(b) \wedge \mathfrak{c}(v)$.

(5) If $\mathfrak{p}(a) \vee \mathfrak{p}(b)=1$, then there exist $u, v \in L$ such that $\mathfrak{p}(u) \wedge \mathfrak{p}(v)=0$ and $\mathfrak{D}(a) \vee \mathfrak{p}(u)=1=$ $\mathfrak{o}(b) \vee \mathfrak{o}(v)$.

(6) If $a \wedge b=0$, with $a$ and $b$ regular, then there exist $u, v \in L$ such that $u \vee v=1$ and $a \wedge u=0=b \wedge v$.

(7) If $\mathfrak{v}(a) \vee \mathfrak{v}(b)=1$, then $\overline{\mathrm{D}(a)} \vee \overline{\mathfrak{p}(b)}=1$.

(8) If $\mathfrak{c}(a) \wedge \mathfrak{c}(b)=0$, then $\mathfrak{c}(a)^{\circ} \wedge \mathfrak{c}(b)^{\circ}=0$.

(9) The interior of every closed sublocale of $L$ is clopen.

(10) The closure of every open sublocale of $L$ is clopen.

(11) The frame $\downarrow$ a is extremally disconnected for every $a \in L$.

Proof. (1) $\Rightarrow(2)$ by Proposition 1.4(5).

(2) $\Rightarrow(3)$ : If $a \wedge b=0$, taking $u=a^{*}$ and $v=b^{*}$ we have that $u \vee v=a^{*} \vee b^{*}=(a \wedge b)^{*}=1$ and $a \wedge u=0=b \wedge v$.

(3) $\Leftrightarrow(4) \Leftrightarrow(5)$ and $(5) \Rightarrow(6)$ are trivial.

(6) $\Rightarrow(1)$ : Since $a^{* *} \wedge a^{*}=0$ and both $a^{* *}$ and $a^{*}$ are regular, there exist $u, v \in L$ satisfying $u \vee v=1$ and $a^{* *} \wedge u=0=v \wedge a^{*}$. This implies that $1=u \vee v \leq a^{* * *} \vee a^{* *}=a^{*} \vee a^{* *}$.

$(2) \Rightarrow(7): \mathfrak{o}(a) \vee \mathfrak{o}(b)=1 \Leftrightarrow a \wedge b=0 \Rightarrow 1=(a \wedge b)^{*}=a^{*} \vee b^{*} \Rightarrow 1=\mathfrak{c}\left(a^{*}\right) \vee \mathfrak{c}\left(b^{*}\right)=\overline{\mathfrak{D}(a)} \vee \overline{\mathfrak{D}(b)}$ (7) $\Leftrightarrow(8)$ : It is obvious, since by complementation, $\mathfrak{D}(a) \vee \mathfrak{p}(b)=1 \Leftrightarrow \mathfrak{c}(a) \wedge \mathfrak{c}(b)=0$ and $\overline{\mathfrak{p}(a)} \vee \overline{\mathrm{D}(b)}=1 \Leftrightarrow \mathfrak{c}(a)^{\circ} \wedge \mathfrak{c}(b)^{\circ}=0$.

$(8) \Rightarrow(9)$ : Since $\mathfrak{c}(a) \wedge \mathfrak{c}\left(a^{*}\right)=0$, it follows that $0=\mathfrak{c}(a)^{\circ} \wedge \mathfrak{c}\left(a^{*}\right)^{\circ}=\mathfrak{v}\left(a^{*}\right) \wedge \mathfrak{p}\left(a^{* *}\right)$ and so $\mathfrak{p}\left(a^{*}\right)=\mathfrak{c}\left(a^{* *}\right)$. Hence, for every closed sublocale $\mathfrak{c}(a), \mathfrak{c}(a)^{\circ}=\mathfrak{v}\left(a^{*}\right)=\mathfrak{c}\left(a^{* *}\right)$.

$(9) \Rightarrow(10)$ : Consider an open sublocale $\mathfrak{D}(a)$. Then, by hypothesis $\mathfrak{c}(a)^{\circ}$ is clopen and thus, by Proposition 1.1(3), $\overline{\mathrm{D}(a)}=\left(\mathfrak{c}(a)^{\circ}\right)^{\mathrm{c}}$ is also clopen.

$(10) \Rightarrow(1)$ : Let $a \in L$. Since $\overline{\mathrm{D}(a)}=\mathfrak{c}\left(a^{*}\right)$ is clopen it follows that $a^{*}$ is complemented in $L$.

$(1) \Leftrightarrow(11)$ was proved in [15, Remark 5.4(2)]. 
Remark 2.4. The infinite version of the first De Morgan law, $\left(\bigvee_{I} a_{i}\right)^{*}=\bigwedge_{I} a_{i}^{*}$ (for any index set $I)$, holds in every frame. However, contrarily to what we incorrectly wrote in [21, pg. 2270], the dual law $\left(\bigwedge_{i \in I} a_{i}\right)^{*}=\bigvee_{i \in I} a_{i}^{*}$ does not hold in general for extremally disconnected frames.

As a first step towards establishing our general setting, note that conditions 2.1 and 2.3(3) that characterize normal and extremally disconnected frames respectively are dual to each other. They are formulable in any lattice and so one may speak more generally about normal and extremally disconnected lattices (this idea is not new in the literature, see e.g. [48, Section 82] and the nice discussion there about the duality between normal and extremally disconnected distributive lattices). Evidently, a lattice $L$ is normal iff its dual lattice $L^{o p}$ is extremally disconnected. Moreover, for frames the following is also obvious:

Corollary 2.5. Let L be a frame. Then:

(a) $L$ is normal iff $\mathfrak{c}(L)$ is normal iff $\mathfrak{D}(L)$ is extremally disconnected.

(b) $L$ is extremally disconnected iff $\mathfrak{c}(L)$ is extremally disconnected iff $\mathrm{p}(L)$ is normal.

We shall now consider the counterparts in the pointfree setting of some other notions related to normality that one finds in the literature. All these examples will be of interest later on.

We say that a frame $L$ is mildly normal (resp. almost normal) if for any $a, b \in L$ satisfying $a \vee b=1$, with $a$ and $b$ (resp. $a$ ) regular, there exist $u, v \in L$ such that $u \wedge v=0$ and $a \vee u=b \vee v=1$. They are the frame counterparts of mildly normal spaces [43] and almost normal spaces [42].

Remark 2.6. By 2.3(6) (which relies on the equivalence $a \wedge b=0 \Leftrightarrow a^{* *} \wedge b^{* *}=0$ of Proposition 1.4), the corresponding notions in the extremally disconnected side do not give anything really weaker. The difference with normality is that the dual equivalence

$$
a \vee b=1 \Leftrightarrow a^{* *} \vee b^{* *}=1
$$

does not hold in arbitrary distributive $p$-algebras.

Finally, we also need to introduce the following definitions:

A sublocale $S$ is a regular $G_{\delta}$ sublocale in case $S$ is a countable join of closed sublocales $c\left(a_{n}\right)$ whose interiors are contained in $S$, that is,

$$
S=\bigvee_{n} \mathfrak{c}\left(a_{n}\right)=\bigvee_{n} \mathfrak{c}\left(a_{n}\right)^{\circ}=\bigvee_{n} \mathfrak{p}\left(a_{n}{ }^{*}\right)
$$

Dually, $S$ is a regular $F_{\sigma}$ sublocale in case

$$
S=\bigwedge_{n} \mathfrak{p}\left(a_{n}\right)=\bigwedge_{n} \overline{\mathfrak{p}\left(a_{n}\right)}=\bigwedge_{n} \mathfrak{c}\left(a_{n}^{*}\right)
$$

An element $a$ of $L$ is $\delta$-regular whenever $a=\bigvee_{n} a_{n}$ with $a_{n}<a$. Regarding these notions we have:

$$
a \delta \text {-regular } \Longleftrightarrow \mathrm{c}(a) \text { regular } G_{\delta} \text { sublocale } \Longrightarrow \mathfrak{D}(a) \text { regular } F_{\sigma} \text { sublocale. }
$$

(The equivalence is an immediate consequence of the fact that $a_{n} \prec a$ iff $\mathfrak{o}\left(a_{n}{ }^{*}\right) \leq \mathfrak{c}(a)$ : if $a$ is $\delta$-regular then $\mathfrak{c}(a)=\bigvee_{n} \mathfrak{c}\left(a_{n}\right) \leq \bigvee_{n} \mathfrak{c}\left(a_{n}\right)^{\circ}=\bigvee_{n} \mathfrak{v}\left(a_{n}{ }^{*}\right) \leq \mathfrak{c}(a)$ thus $\mathfrak{c}(a)=\bigvee_{n} \mathfrak{v}\left(a_{n}{ }^{*}\right)$; the converse follows similarly. The second implication is obvious while its converse is false by a counterexample in [18].)

We say that a frame is $\delta$-normal if for any $a, b \in L$ satisfying $a \vee b=1$, with $a$ and $b \delta$ regular, there exist $\delta$-regular elements $u, v \in L$ such that $u \wedge v=0$ and $a \vee u=b \vee v=1$. For any 
topological space $X$, the $\delta$-regular elements of the frame $O X$ of open subsets of $X$ consist exactly of the regular $F_{\sigma}$ subsets of $X$ (the complements of the usual regular $G_{\delta}$ subsets of $X$ [37]), and therefore $\delta$-normal frames extend $\delta$-normal spaces.

Remark 2.7. In the definition above of a $\delta$-regular element we may assume that each $a_{n}$ is regular. Indeed, $a_{n} \prec a$ implies $a_{n}{ }^{* *}<a$ and hence $a=\bigvee_{n} a_{n} \leq \bigvee_{n} a_{n}{ }^{* *} \leq a$.

\section{Variants of semicontinuity}

Recall from [17] that a real function $f$ on $L$ is:

(1) lower semicontinuous if $f(r,-)$ is a closed sublocale for every $r \in \mathbb{Q}$;

(2) upper semicontinuous if $f(-, r)$ is a closed sublocale for every $r \in \mathbb{Q}$;

(3) continuous if $f(p, q)$ is a closed sublocale for every $p, q$, i.e. $f(\mathfrak{L}(\mathbb{R})) \subseteq \mathfrak{c}(L)$.

We denote by

$$
\operatorname{LSC}(L), \operatorname{USC}(L) \text { and } \mathrm{C}(L)
$$

the collections of all lower semicontinuous, upper semicontinuous, and continuous members of $\mathrm{F}(L)$. Note that if $S$ is a complemented sublocale of $L$, then $\chi_{S} \in \operatorname{LSC}(L)$ iff $S$ is open and dually $\chi_{S} \in \operatorname{USC}(L)$ iff $S$ is closed. It is also worth mention that, as proved in [17], after the isomorphism $\mathfrak{c}: L \rightarrow \mathfrak{c}(L)$, the elements of $\mathrm{C}(L)$ are represented by frame homomorphisms $\varphi: \mathfrak{L}(\mathbb{R}) \rightarrow L$, thus coinciding with the standard continuous real functions on a frame $L$ (see [3]). In the sequel, we will freely refer to a continuous real function as both the real function $f \in \mathrm{C}(L)$ and the unique frame homomorphism $\varphi: \mathfrak{L}(\mathbb{R}) \rightarrow L$ such that $\mathfrak{c} \cdot \varphi=f$.

Proposition 3.1. Let $f \in \mathrm{F}(L)$. Then:

(1) $f \in \operatorname{LSC}(L)$ if and only if for each $p<q$ in $\mathbb{Q}$ there exists a closed sublocale $F_{p, q}$ such that $f(q,-) \leq F_{p, q} \leq f(p,-)$.

(2) $f \in \operatorname{USC}(L)$ if and only if for each $p<q$ in $\mathbb{Q}$ there exists a closed sublocale $F_{p, q}$ such that $f(-, p) \leq F_{p, q} \leq f(-, q)$.

Proof. (1) $\Rightarrow$ : This is obvious since $f(p,-)$ is closed for each $p \in \mathbb{Q}$.

$\Leftarrow$ : Let $p \in \mathbb{Q}$. Then $f(p,-)=\bigvee_{r>p} f(r,-) \leq \bigvee_{r>p} F_{p, r} \leq f(p,-)$ and thus $f(p,-)=\bigvee_{r>p} F_{p, r}$ is a closed sublocale.

(2) Similar to (1).

An element $a \in L$ is said to be a cozero element if there exists an $f \in \mathrm{C}(L)$ such that

$$
\mathfrak{c}(a)=f((-, 0) \vee(0,-))=\bigvee\{f(p, 0) \vee f(0, q) \mid p<0<q \text { in } \mathbb{Q}\},
$$

or, equivalently, a continuous $\varphi: \mathfrak{Q}(\mathbb{R}) \rightarrow L$ such that $a=\varphi((-, 0) \vee(0,-))$. We shall denote $a$ by $\operatorname{coz} f$.

Remarks 3.2. (1) The closed sublocales $\mathfrak{c}(a)$ given by cozero elements $a=\operatorname{coz} f$ are the zero-set sublocales of [26], complements of the cozero sublocales $\mathfrak{v}(\operatorname{coz} f)$.

(2) The cozero elements can be alternatively described without reference to the frame of reals as follows [5]: $a$ is a cozero element iff $a=\bigvee_{n} a_{n}$ for some $a_{n} \ll a, n=1,2, \ldots$ (equivalently: $\left.a_{n} \prec<a_{n+1}, n=1,2, \ldots\right)$. 
(3) For more information on the map coz: $\mathrm{C}(L) \rightarrow L$ we refer to [4]. As usual, Coz $L$ will denote the cozero lattice of all cozero elements of $L$ (which is always a sub- $\sigma$-frame of $L$ by (2)). Note that in any extremally disconnected frame, each regular element $a$, being complemented, is a cozero element (indeed, $\chi_{\mathrm{v}(a)} \in \mathrm{C}(L)$ and $\chi_{\mathfrak{v}(a)}((-, 0) \vee(0,-))=\mathfrak{c}(a)$, hence $\left.a=\operatorname{coz} \chi_{\mathfrak{v}(a)}\right)$.

(4) It is easy to see that cozero elements are $\delta$-regular $\left(\right.$ since $\varphi((-, 0) \vee(0,-))=\bigvee_{n} \varphi\left(-\frac{1}{n}, \frac{1}{n}\right)^{*}$ for any continuous $\varphi: \mathfrak{L}(\mathbb{R}) \rightarrow L)$. On the other hand, in any almost normal frame, each $\delta$-regular element belongs to $\operatorname{Coz} L$. In fact, for $a=\bigvee_{n} a_{n}$ with $a_{n}<a$ and $a_{n}$ regular, we have $a_{n}{ }^{*} \vee a=1$, and so by almost normality there exist $u_{n}$ and $v_{n}$ such that $a_{n}{ }^{*} \vee u_{n}=1$ and $u_{n}{ }^{*} \vee a \geq v_{n} \vee a=1$, which means that $a_{n} \prec u_{n} \prec a$, and thus $a_{n} \prec<a$ (by Remark 2.2).

The lower and upper regularizations of $f$ were introduced and studied in [15, 17]. In general they are extended real functions $[17,6]$ but if there exists $g \in \operatorname{LSC}(L)$ such that $g \leq f$, the lower regularization $f^{\circ}$ of $f$ is a real function, generated by the scale $(\overline{f(r,-)} \mid r \in \mathbb{Q})$, i.e.,

$$
f^{\circ}(p,-)=\bigvee_{r>p} \overline{f(r,-)} \quad \text { and } \quad f^{\circ}(-, q)=\bigvee_{s<q}(\overline{f(s,-)})^{\mathrm{c}}
$$

Dually, if there exists $g \in \operatorname{USC}(L)$ such that $f \leq g$, the upper regularization $f^{-}$is the real function generated by the scale $\left((\overline{f(-, r)})^{\mathrm{c}} \mid r \in \mathbb{Q}\right)$, i.e.,

$$
f^{-}(p,-)=\bigvee_{r>p}(\overline{f(-, r)})^{c} \quad \text { and } \quad f^{-}(-, q)=\bigvee_{s<q} \overline{f(-, s)}
$$

Given an $f \in \mathrm{F}(L)$, the function $-f$ is defined by $(-f)(p,-)=f(-,-p)$ and $(-f)(-, q)=$ $f(-q,-)$ for every $p, q \in \mathbb{Q}$. Note that if there exist $g_{1} \in \operatorname{LSC}(L)$ and $g_{2} \in \operatorname{USC}(L)$ such that $g_{1} \leq f \leq g_{2}$ then $(-f)^{\circ}=-f^{-},(-f)^{-}=-f^{\circ},(-f)^{\circ-}=-f^{-\circ}$ and $(-f)^{-\circ}=-f^{\circ-}$.

Let

$$
\begin{aligned}
& \mathrm{F}^{b l}(L)=\{f \in \mathrm{F}(L) \mid \exists g \in \operatorname{LSC}(L) \text { such that } g \leq f\}, \\
& \mathrm{F}^{b u}(L)=\{f \in \mathrm{F}(L) \mid \exists g \in \operatorname{USC}(L) \text { such that } f \leq g\} \text { and } \\
& \mathrm{F}^{b}(L)=\mathrm{F}^{b l}(L) \cap \mathrm{F}^{b u}(L) .
\end{aligned}
$$

The operators $(\cdot)^{\circ}: \mathrm{F}^{b l}(L) \rightarrow \operatorname{LSC}(L)$ and $(\cdot)^{-}: \mathrm{F}^{b u}(L) \rightarrow \operatorname{USC}(L)$ have the following useful properties $([15,17])$ :

Proposition 3.3. Let $f, f_{1}, f_{2} \in \mathrm{F}^{b l}(L)$ and $g, g_{1}, g_{2} \in \mathrm{F}^{b u}(L)$. Then:

(1) $f^{\circ} \leq f$ and $g \leq g^{-}$.

(2) $f^{\circ \circ}=f^{\circ}$ and $f^{--}=f^{-}$.

(3) $\left(f_{1} \wedge f_{2}\right)^{\circ}=f_{1}^{\circ} \wedge f_{2}^{\circ}$ and $\left(g_{1} \vee g_{2}\right)^{-}=g_{1}{ }^{-} \vee g_{2}{ }^{-}$.

(4) $\operatorname{LSC}(L)=\left\{f \in \mathrm{F}^{b l}(L) \mid f^{\circ}=f\right\}$ and $\operatorname{USC}(L)=\left\{f \in \mathrm{F}^{b u}(L) \mid f^{-}=f\right\}$.

(5) $f^{\circ}=\bigvee\{g \in \operatorname{LSC}(L) \mid g \leq f\}$ and $f^{-}=\bigwedge\{g \in \operatorname{USC}(L) \mid g \geq f\}$.

(6) For each complemented sublocale $S,\left(\chi_{S}\right)^{\circ}=\chi_{S^{\circ}}$ and $\left(\chi_{S}\right)^{-}=\chi_{\bar{S}}$.

Furthermore:

Lemma 3.4. Let $f \in \mathrm{F}^{b}(L)$. Then, for every $p, q \in \mathbb{Q}$ we have:

(1) $f^{-\circ}(p,-)=\bigvee_{r>p} \overline{f(r,-)^{\circ}}$ and $f^{-\circ}(-, q)=\bigvee_{s<q}(\overline{f(-, s)})^{\circ}$.

(2) $f^{\circ-}(p,-)=\bigvee_{r>p}(\overline{f(r,-)})^{\circ}$ and $f^{\circ-}(-, q)=\bigvee_{s<q} \overline{f(-, s)^{\circ}}$. 
Proof. (1) First note that since $f \in \mathrm{F}^{b}(L)$, it follows that $f^{-} \in \mathrm{F}^{b l}(L)$ and so $f^{-\circ} \in \mathrm{F}(L)$. By definition, for each $p \in \mathbb{Q}$,

$$
f^{-\circ}(p,-)=\bigvee_{r>p} \overline{f^{-(}(r,-)}=\underset{r>p}{\bigvee} \overline{\bigvee_{s>r}(\overline{f(-, s)})^{\mathrm{c}}} .
$$

Let $s>r>p$. Then $f(-, s) \geq f(r,-)^{*}$ and so $(\overline{f(-, s)})^{\mathrm{c}} \leq\left(\overline{f(r,-)^{*}}\right)^{\mathrm{c}}$. It follows that $\bigvee_{s>r}(\overline{f(-, s)})^{\mathrm{c}} \leq\left(\overline{f(r,-)^{*}}\right)^{\mathrm{c}}=f(r,-)^{\circ}$ and thus

$$
f^{-o}(p,-)=\bigvee_{r>p} \overline{\bigvee_{s>r}(\overline{f(-, s)})^{\mathrm{c}}} \leq \bigvee_{r>p} \overline{f(r,-)^{\circ}} .
$$

Conversely, let $r>r^{\prime}>p$. Then $f(-, r) \leq f(r,-)^{*}$ and so

$$
f(r,-)^{\circ}=\left(\overline{f(r,-)^{*}}\right)^{\mathrm{c}} \leq(\overline{f(-, r)})^{\mathrm{c}} \leq \bigvee_{s>r^{\prime}}(\overline{f(-, s)})^{\mathrm{c}} .
$$

Hence $\overline{f(r,-)^{\circ}} \leq \overline{\bigvee_{s>r^{\prime}}(\overline{f(-, s)})^{c}}$ and therefore

$$
\bigvee_{r>p} \overline{f(r,-)^{\circ}}=\bigvee_{r^{\prime}>p} \bigvee_{r>r^{\prime}} \overline{f(r,-)^{\circ}} \leq \bigvee_{r^{\prime}>p} \overline{\bigvee_{s>r^{\prime}}(\overline{f(-, s)})^{\mathrm{c}}}=f^{-\circ}(p,-) .
$$

On the other hand, for each $q \in \mathbb{Q}$ we have (again by definition)

$$
f^{-\circ}(-, q)=\bigvee_{s<q}\left(\overline{f^{-}(s,-)}\right)^{\mathrm{c}}=\bigvee_{s<q}\left(\overline{\bigvee_{r>s}(\overline{f(-, r)})^{c}}\right)^{\mathrm{c}} .
$$

Let $s<t<q$. Then $\bigvee_{r>s}(\overline{f(-, r)})^{\mathrm{c}} \geq(\overline{f(-, t)})^{\mathrm{c}}$ and so

$$
\left(\overline{\bigvee_{r>s}(\overline{f(-, r)})^{c}}\right)^{c} \leq\left(\overline{(\overline{f(-, t)})^{c}}\right)^{c}=(\overline{f(-, t)})^{\circ}
$$

It follows that

$$
f^{-\circ}(-, q)=\bigvee_{s<q}\left(\overline{\bigvee_{r>s}(\overline{f(-, r)})^{\mathrm{c}}}\right)^{\mathrm{c}} \leq \bigvee_{t<q}(\overline{f(-, t)})^{\circ} .
$$

Conversely, let $s<q$. Then $f(-, s) \leq f(-, r)$ for all $r>s$ and so

$$
(\overline{f(-, s)})^{\mathrm{c}} \geq \bigvee_{r>s}(\overline{f(-, r)})^{\mathrm{c}} \text {. }
$$

Hence

$$
\bigvee_{s<q}(\overline{f(-, s)})^{\circ}=\bigvee_{s<q}\left(\overline{(\overline{f(-, s)})^{c}}\right)^{c} \leq \bigvee_{s<q}\left(\overline{\bigvee_{r>s}(\overline{f(-, r)})^{c}}\right)^{c}=f^{-\circ}(-, q) .
$$

(2) It follows immediately since $f^{\circ-} \in \mathrm{F}(L)$ and

$$
\begin{aligned}
f^{\circ-}(p,-) & =-\left(f^{\circ-}\right)(-,-p)=(-f)^{-\circ}(-,-p) \\
& =\bigvee_{s<-p}(\overline{(-f)(-, s)})^{\circ}=\bigvee_{s<-p}(\overline{f(-s,-)})^{\circ}=\bigvee_{r>q}(\overline{f(r,-)})^{\circ}
\end{aligned}
$$

and

$$
\begin{aligned}
f^{\circ-}(-, q) & =-\left(f^{\circ-}\right)(-q,-)=(-f)^{-\circ}(-q,-) \\
& =\underset{r>-q}{\bigvee} \overline{(-f)(r,-)^{\circ}}=\bigvee_{r>-q} \overline{f(-,-r)^{\circ}}=\bigvee_{s<q} \overline{f(-, s)^{\circ}} .
\end{aligned}
$$


In the point-set case, one finds in the literature several special notions of lower and upper semicontinuity (see [10, 35, 36, 44]). Normal lower and upper semicontinuous functions, introduced by Dilworth ([10]), are immediately extendable to frames: we say that an $f \in \mathrm{F}(L)$ is normal upper (resp. normal lower) semicontinuous if $\left(f^{\circ}\right)^{-}=f$ (resp. $\left(f^{-}\right)^{\circ}=f$ ). Note that if $f$ is normal upper or lower semicontinuous, then $f \in \mathrm{F}^{b}(L)$. It follows immediately from Lemma 3.4 that:

Corollary 3.5. Let $f \in \mathrm{F}^{b}(L)$. Then:

(1) $f$ is normal lower semicontinuous if and only if $f(p,-)=\bigvee_{r>p} \overline{f(r,-)^{\circ}}$ for every $p \in \mathbb{Q}$. Denoting by $a_{p}$ the element in L such that $f(p,-)=\mathfrak{c}\left(a_{p}\right)$, then $f$ is normal lower semicontinuous if and only if $a_{r}=\bigvee_{r>p} a_{r}{ }^{* *}$ for every $p \in \mathbb{Q}$.

(2) $f$ is normal upper semicontinuous if and only if $f(-, q)=\bigvee_{s<q} \overline{f(-, s)^{\circ}}$ for every $q \in \mathbb{Q}$. Denoting by $b_{q}$ the element in L such that $f(-, q)=\mathrm{c}\left(b_{q}\right)$, then $f$ is normal upper semicontinuous if and only if $b_{q}=\bigvee_{s<q} b_{s}^{* *}$ for every $q \in \mathbb{Q}$.

Now we may characterize normal semicontinuity in a similar vein to Proposition 3.1, as follows:

Proposition 3.6. Let $f \in \mathrm{F}^{b}(L)$. Then:

(1) $f$ is normal lower semicontinuous if and only if for each $p<q$ in $\mathbb{Q}$ there exists a regular closed sublocale $F_{p, q}$ such that $f(q,-) \leq F_{p, q} \leq f(p,-)$.

(2) $f$ is normal upper semicontinuous if and only if for each $p<q$ in $\mathbb{Q}$ there exists a regular closed sublocale $F_{p, q}$ such that $f(-, p) \leq F_{p, q} \leq f(-, q)$.

Proof. (1) $\Rightarrow$ : Let $p<q$. Then for each $r>q, f(r,-) \leq f(q,-)$ and thus

$$
f^{-\circ}(q,-)=\bigvee_{r>q} \overline{f(r,-)^{\circ}} \leq \overline{f(q,-)^{\circ}} .
$$

Hence

$$
f(q,-)=f^{-\circ}(q,-) \leq \overline{f(q,-)^{\circ}} \leq \bigvee_{r>p} \overline{f(r,-)^{\circ}} \leq f^{-\circ}(p,-)=f(p,-),
$$

with $\overline{f(q,-)^{\circ}}$ being a regular closed sublocale.

$\Leftarrow$ : We first note that since $F_{p, q}$ is closed for each $p<q$, it follows from Proposition 3.1 that $f$ is lower semicontinuous. Hence $f=f^{\circ} \leq f^{-\circ}$. On the other hand, let $p \in \mathbb{Q}$. Then for each $r>p$ there exists a regular closed sublocale $F_{p, r}$ such that $f(r,-) \leq F_{p, r} \leq f(p,-)$. Therefore $\overline{f(r,-)^{\circ}} \leq \overline{F_{p, r}^{\circ}}=F_{p, r} \leq f(p,-)$ and hence

$$
f^{-\circ}(p,-)=\bigvee_{r>p} \overline{f(r,-)^{\circ}} \leq \bigvee_{r>p} F_{p, r} \leq f(p,-)
$$

It follows that $f^{-\circ} \leq f$.

(2) Similar to (1).

Let $S$ be a closed sublocale of $L$. Then $\chi_{S} \in \operatorname{USC}(L)$ and it is normal if and only if $\overline{S^{\circ}}=S$, that is, if and only if $S$ is a regular closed sublocale. In case $L$ is extremally disconnected (which means that $\overline{S^{\circ}}=S^{\circ}$ for every closed $S$ ) then $S$ is clopen. Thus every normal upper $\chi_{S}$ is continuous if and only if $L$ is extremally disconnected. More generally, we have: 
Corollary 3.7. The following are equivalent for any frame $L$ :

(1) L is extremally disconnected.

(2) Every normal lower semicontinuous function on L is continuous.

(3) Every normal upper semicontinuous function on $L$ is continuous.

Proof. The implications "(2) $\Leftrightarrow(3) \Rightarrow(1)$ " are obvious. Regarding (1) $\Rightarrow(2)$, let $L$ be extremally disconnected and consider $f \in \operatorname{LSC}(L)$ such that $f^{-\circ}=f$. Then, for each $r<t<s$ in Q,

$$
\begin{aligned}
f^{-}(-, s) \vee f(r,-) & =f^{-}(-, s) \vee f^{-\circ}(r,-) \geq\left(\vee_{q<s} \overline{f(-, q)}\right) \vee\left(\vee_{p>r} \overline{f(p,-)^{\circ}}\right) \\
& \geq \overline{f(t,-)^{*}} \vee \overline{f(t,-)^{\circ}}=\mathfrak{c}\left(a_{t}^{*}\right) \vee \mathfrak{c}\left(a_{t}^{* *}\right)=1
\end{aligned}
$$

Consequently, $f^{-}(-, s) \geq \bigvee_{r<s} f(r,-)^{*} \geq \bigvee_{r<s} f(-, r)=f(-, s)$. This means that $f^{-} \leq f$ and therefore $f=f^{-} \in \operatorname{USC}(L)$. Hence $f$ is continuous.

Other important classes of classical semicontinuous functions are the zero ones of Stone [44] and the regular ones of Lane [36]. Regarding the former, we say that an $f \in \mathrm{F}(L)$ is zero lower (resp. zero upper) semicontinuous function if, for each $r \in \mathbb{Q}, f(r,-)=\mathfrak{c}\left(a_{r}\right)$ (resp. $f(-, r)=$ $\left.\mathfrak{c}\left(a_{r}\right)\right)$ for some $a_{r} \in \operatorname{Coz} L$. Since $\operatorname{Coz} L$ is a sub- $\sigma$-frame of $L$, this is equivalent to saying that for each $p<q$ in $\mathbb{Q}$ there exists a zero-set sublocale $\mathfrak{c}\left(a_{p, q}\right)$ such that $f(q,-) \leq \mathfrak{c}\left(a_{p, q}\right) \leq f(p,-)$ (resp. $\left.f(-, p) \leq \mathfrak{c}\left(a_{p, q}\right) \leq f(-, q)\right)$ (recall the proof of Proposition 3.1).

On the other hand, we say that an $f \in \mathrm{F}(L)$ is regular lower (resp. regular upper) semicontinuous if, for each $r \in \mathbb{Q}, f(r,-)=\mathfrak{c}\left(a_{r}\right)$ (resp. $\left.f(-, r)=\mathfrak{c}\left(a_{r}\right)\right)$ where each $a_{r}$ is a $\delta$-regular element. Again, since the $\delta$-regular elements form a sub- $\sigma$-frame of $L$, this is equivalent to saying that for each $p<q$ in $\mathbb{Q}$ there exists a regular closed $G_{\delta}$ sublocale $\mathfrak{c}\left(a_{p, q}\right)$ such that $f(q,-) \leq \mathfrak{c}\left(a_{p, q}\right) \leq f(p,-)$ (resp. $\left.f(-, p) \leq \mathfrak{c}\left(a_{p, q}\right) \leq f(-, q)\right)$

Note that zero semicontinuity implies regular semicontinuity; by Remark 3.2(4), the converse holds in almost normal frames.

Finally, recall from [22] (see also [13]) that an $f \in \mathrm{F}(L)$ is lower (resp. upper) compact-like if $f(r,-)$ (resp. $f(-, r)$ ) is a compact sublocale of $L$ for every $r \in \mathbb{Q}$. In any Hausdorff (or fit) frame, compact sublocales are closed. In that case, therefore, any lower (resp. upper) compactlike function on $L$ is lower (resp. upper) semicontinuous. It should be added that if $L$ is compact, then any upper (resp. lower) semicontinuous function on $L$ is upper (resp. lower) compact-like [22].

\section{4. $\mathscr{A}$-normality and $\mathscr{A}$-disconnectedness}

The main idea of our approach is the following: go to the sublocale frame $\mathcal{S}(L)$ and take complements inside it. More specifically, given a frame $L$, let $B(\mathcal{S}(L))$ denote the Boolean part of $\mathcal{S}(L)$ (that is, the Boolean algebra of complemented elements of $\mathcal{S}(L)$ ). Fix an $\mathscr{A} \subseteq B(\mathcal{S}(L)$ ) and let $\mathscr{A}^{\mathrm{c}}$ denote the corresponding set of complements. Clearly, $\mathscr{A}^{\mathrm{cc}}=\mathscr{A}$ and if $\mathscr{A}$ is a sublattice of $B(\mathcal{S}(L))$, then $\mathscr{A}^{\mathrm{c}}$ is also a sublattice of $B(\mathcal{S}(L))$.

Definition 4.1. A frame $L$ is $\mathscr{A}$-normal if

for any $A, B \in \mathscr{A}$ such that $A \vee B=1$ there are $U, V \in \mathscr{A}$ such that $U \wedge V=0$ and $A \vee U=1=B \vee V$. 
Dually, we say that $L$ is $\mathscr{A}$-disconnected if it is $\mathscr{A}^{\mathrm{c}}$-normal, that is, if

for any $A, B \in \mathscr{A}$ such that $A \wedge B=0$ there are $U, V \in \mathscr{A}$ such that $U \vee V=1$

and $A \wedge U=0=B \wedge V$.

Remark 4.2. Note that in case $L$ is $O X$ for some space $X$, since every complemented sublocale of a space is a subspace [40], these notions are completely formulated in terms of the space $X$ (with no reference to sublocales) and provide a unification of several variants of normal and disconnected topological spaces in the literature.

Examples 4.3. Our guiding examples for classes $\mathscr{A} \subseteq B(\mathcal{S}(L))$ will be

$$
\begin{array}{ll}
\mathscr{A}_{1}=\mathfrak{c}(L), & \mathscr{A}_{2}=\left\{\mathfrak{c}\left(a^{*}\right) \mid a \in L\right\}, \\
\mathscr{A}_{4}=B(\mathcal{S}(L)), & \mathscr{A}_{5}=\{\mathfrak{c}(a) \mid a \text { is } \delta \text {-regular }\} .
\end{array}
$$

$\mathscr{A}_{1}, \mathscr{A}_{3}, \mathscr{A}_{4}$ and $\mathscr{A}_{5}$ are clearly sublattices of $B(\mathcal{S}(L))$ while $\mathscr{A}_{2}$ is only closed under finite meets. They induce the notions listed in Table 3.

\begin{tabular}{lll}
\hline $\mathscr{A}$ & $\mathscr{A}$-normal frames & $\mathscr{A}$-disconnected frames \\
\hline $\mathscr{A}_{1}$ & normal frames & extremally disconnected frames \\
$\mathscr{A}_{2}$ & mildly normal frames & extremally disconnected frames \\
$\mathscr{A}_{3}$ & frames & $F$-frames \\
$\mathscr{A}_{4}$ & frames & frames \\
$\mathscr{A}_{5}$ & $\delta$-normal frames & extremally $\delta$-disconnected frames \\
\hline
\end{tabular}

Table 3: Examples of $\mathscr{A}$-normal and $\mathscr{A}$-disconnected frames

Indeed, the cases $\mathscr{A}_{1}$ and $\mathscr{A}_{4}$ are obvious. Concerning $\mathscr{A}_{2}$ we have:

- $\mathscr{A}_{2}$-normal=mildly normal: if $a \vee b=1$ (with $a, b$ regular elements) and there are $u, v \in L$ such that $u \wedge v=0$ and $a \vee u=1=b \vee v$, then $a \vee u^{* *}=1=b \vee v^{* *}$ and again $u^{* *} \wedge v^{* *}=0$ (by Proposition 1.4(1)).

- $\mathscr{A}_{2}$-disconnected=extremally disconnected: Proposition 2.3(6) (cf. Remark 2.6).

For $\mathscr{A}_{3}$ (the zero-set sublocales of Remark 3.2(1)), the fact that any frame is $\mathscr{A}_{3}$-normal is a consequence of Proposition 5 in Banaschewski ([2]) while the fact that $\mathscr{A}_{3}$-disconnected frames are precisely the $F$-frames (i.e, frames where the open quotient of each (dense) cozero element is a $C^{*}$-quotient) follows from Proposition 8.4.10 in Ball and Walters-Wayland [1].

Finally, for $\mathscr{A}_{5}$, it is obvious that the $\mathscr{A}_{5}$-normal frames are what we named $\delta$-normal frames; accordingly, we call $\mathscr{A}_{5}$-disconnected frames as extremally $\delta$-disconnected frames. Note that by Proposition 8.4 .10 of [1], in frames where the $\delta$-regular elements are the cozero elements, extremally $\delta$-disconnected frames coincide with $F$-frames.

\section{5. $\mathscr{A}$-continuity and $\mathscr{A}$-semicontinuities}

In this section we show how variants of continuous and semicontinuous real functions can also be defined in terms of a given class of complemented sublocales $\mathscr{A}$. 
Ordinary continuity and semicontinuities of an $f: \mathfrak{L}(\mathbb{R}) \rightarrow \mathcal{S}(L)$ are defined with respect to $\mathfrak{c}(L) \subseteq \mathcal{S}(L)$. In this case, by Proposition 3.1, $f$ is lower (resp. upper) semicontinuous if and only if for each $p<q$ in $\mathbb{Q}$ there exists $F_{p, q} \in \mathfrak{c}(L)$ such that $f(q,-) \leq F_{p, q} \leq f(p,-)$ (resp. $\left.f(-, p) \leq F_{p, q} \leq f(-, q)\right)$. This is so because $\mathfrak{c}(L)$ is closed under joins. However, for a general class $\mathscr{A}$ of complemented sublocales not necessarily closed under joins the latter condition is weaker and we take it as the definition for semicontinuity with respect to $\mathscr{A}$.

Definitions 5.1. We say that $f$ is:

(1) lower $\mathscr{A}$-semicontinuous if for each $p<q$ in $\mathbb{Q}$ there exists $F_{p, q} \in \mathscr{A}$ such that $f(q,-) \leq$ $F_{p, q} \leq f(p,-)$.

(2) upper $\mathscr{A}$-semicontinuous if for each $p<q$ in $\mathbb{Q}$ there exists $F_{p, q} \in \mathscr{A}$ such that $f(-, p) \leq$ $F_{p, q} \leq f(-, q)$.

(3) $\mathscr{A}$-continuous if it is both lower and upper $\mathscr{A}$-semicontinuous.

Remark 5.2. $f$ is upper $\mathscr{A}$-semicontinuous iff it is lower $\mathscr{A}^{\mathrm{c}}$-semicontinuous (and therefore it is $\mathscr{A}^{\mathrm{C}}$-continuous iff it is $\mathscr{A}$-continuous): if for each $p<q$ in $\mathbb{Q}$ there is some $F_{p, q} \in \mathscr{A}$ satisfying $f(q,-) \leq F_{p, q} \leq f(p,-)$ then take a rational $r$ such that $p<r<q$; since $f(r,-) \leq F_{p, r} \leq f(p,-)$, then $f(-, p) \leq f(p,-)^{*} \leq F_{p, r}{ }^{c}$ and, on the other hand, $f(-, q) \vee F_{p, r} \geq f(-, q) \vee f(r,-)=1$ so that $F_{p, r}{ }^{\mathrm{c}} \leq f(-, q)$.

Note that for any $S \in \mathscr{A}, \chi_{S}$ is upper $\mathscr{A}$-semicontinuous and $\chi_{S^{\circ}}$ is lower $\mathscr{A}$-semicontinuous.

Definition 5.3. (Cf. [14, Def. 3.1]) Further, we say that two sublocales $S$ and $T$ of $L$ are completely $\mathscr{A}$-separated if there is an $\mathscr{A}$-continuous function $f$ such that $f(0,-) \leq S$ and $f(-, 1) \leq T$.

Examples 5.4. For our guiding examples in 4.3 and

$$
\mathscr{A}_{6}=\{S \in B(\mathcal{S}(L)) \mid S \text { is compact }\}
$$

(in this case we assume that $L$ is Hausdorff or fit so that any compact sublocale is complemented) the corresponding induced notions are listed in Table 4 (recall the results of Section 3).

\begin{tabular}{llll}
\hline $\mathscr{A}$ & lower $\mathscr{A}$-semicontinuous & upper $\mathscr{A}$-semicontinuous & $\mathscr{A}$-continuous \\
\hline $\mathscr{A}_{1}$ & lower semicontinuous & upper semicontinuous & continuous \\
$\mathscr{A}_{2}$ & normal lower & normal upper & continuous \\
& semicontinuous & semicontinuous & \\
$\mathscr{A}_{3}$ & zero lower semicontinuous & zero upper semicontinuous & zero continuous \\
$\mathscr{A}_{4}$ & $\mathrm{C}(B(\mathcal{S}(L)))$ & $\mathrm{C}(B(\mathcal{S}(L)))$ & $\mathrm{C}(B(\mathcal{S}(L)))$ \\
$\mathscr{A}_{5}$ & regular lower & regular upper & regular continuous \\
& semicontinuous & semicontinuous & \\
$\mathscr{A}_{6}$ & compact-like lower & compact-like upper & compact-like \\
& semicontinuous & semicontinuous & continuous \\
\hline
\end{tabular}

Table 4: Examples of $\mathscr{A}$-continuous and $\mathscr{A}$-semicontinuous maps

Concerning class $\mathscr{A}_{4}$, since $\mathscr{A}_{4}^{\mathrm{c}}=\mathscr{A}_{4}$, by Remark 5.2 the three classes of induced maps coincide, and are equal to the class of frame homomorphisms $\mathfrak{L}(\mathbb{R}) \rightarrow B(\mathcal{S}(L))$. 


\section{Katětov relations}

Let $M$ be an arbitrary lattice. Recall that a binary relation $\Subset$ on $M$ is called a Katětov relation $([28,30,32,19])$ if it satisfies the following conditions for all $a, b, a^{\prime}, b^{\prime} \in M$ :

(K1) $a \Subset b \Rightarrow a \leq b$.

(K2) $a^{\prime} \leq a \Subset b \leq b^{\prime} \Rightarrow a^{\prime} \Subset b^{\prime}$.

(K3) $a \Subset b$ and $a^{\prime} \Subset b \Rightarrow\left(a \vee a^{\prime}\right) \Subset b$.

(K4) $a \Subset b$ and $a \Subset b^{\prime} \Rightarrow a \Subset\left(b \wedge b^{\prime}\right)$.

(K5) $a \Subset b \Rightarrow \exists c \in M: a \Subset c \Subset b$.

(Interpolation Property)

Lemma 6.1 ([30, 32, 19]). Let $M$ be a lattice, $\Subset$ a Katětov relation on $M$ and $\triangleleft$ a transitive and irreflexive relation on a countable set D. Further, let $\left(a_{d} \mid d \in D\right)$ and $\left(b_{d} \mid d \in D\right)$ be two families of elements of $M$ such that

$$
d_{1} \triangleleft d_{2} \quad \text { implies } \quad a_{d_{2}} \leq a_{d_{1}}, \quad b_{d_{2}} \leq b_{d_{1}} \text { and } a_{d_{2}} \Subset b_{d_{1}} .
$$

Then there exists a family $\left(c_{d} \mid d \in D\right) \subseteq M$ such that

$$
d_{1} \triangleleft d_{2} \quad \text { implies } \quad c_{d_{2}} \Subset c_{d_{1}}, \quad a_{d_{2}} \Subset c_{d_{1}} \text { and } c_{d_{2}} \Subset b_{d_{1}} .
$$

Now, given a fixed $\mathscr{A} \subseteq B(\mathcal{S}(L))$, define the relation $\Subset_{\mathscr{A}}$ on $\mathcal{S}(L)$ by

$$
S \Subset_{\mathscr{A}} T \equiv \exists U \in \mathscr{A}, \exists V \in \mathscr{A}^{\mathrm{c}}: S \leq V \leq U \leq T .
$$

Lemma 6.2. $\Subset_{\mathscr{A}}$ is interpolative if and only if $L$ is $\mathscr{A}$-normal.

Proof. Let $\Subset_{\mathscr{A}}$ satisfy the Interpolation Property and consider $A, B \in \mathscr{A}$ satisfying $A \vee B=1$. Then $A^{\mathrm{c}} \in \mathscr{A}^{\mathrm{c}}$ satisfies $A^{\mathrm{c}} \leq B$. This means that $A^{\mathrm{c}} \Subset_{\mathscr{A}} B$ and by hypothesis there is a $T \in \mathcal{S}(L)$ such that $A^{\mathrm{C}} \Subset_{\mathscr{A}} T \Subset_{\mathscr{A}} B$. Therefore there are $U_{1}, U_{2} \in \mathscr{A}$ and $V_{1}, V_{2} \in \mathscr{A}^{\mathrm{c}}$ such that $A^{\mathrm{c}} \leq V_{1} \leq U_{1} \leq T \leq V_{2} \leq U_{2} \leq B$. Then, immediately, $U_{1} \wedge V_{2}{ }^{\mathrm{C}}=0$ and $A \vee U_{1}=1=B \vee V_{2}{ }^{\mathrm{c}}$.

Conversely, let $S \leq V \leq U \leq T$ for $S, T \in \mathcal{S}(L), U \in \mathscr{A}$ and $V \in \mathscr{A}^{\mathrm{c}}$. Then $V^{\mathrm{c}} \vee U \geq V^{\mathrm{c}} \vee$ $V=1$ thus by the $\mathscr{A}$-normality of $L$, there exist $A, B \in \mathscr{A}$ such that $A \wedge B=0, V^{\mathrm{c}} \vee A=1=U \vee B$. This implies $S \leq V \leq A \leq B^{\mathrm{C}} \leq U \leq T$ which means that $S \Subset_{\mathscr{A}} A \Subset_{\mathscr{A}} T$.

Clearly, for any $\mathscr{A} \subseteq B(\mathcal{S}(L))$, the relation $\Subset_{\mathscr{A}}$ satisfies conditions (K1) and (K2). We say that $\mathscr{A}$ is a Katětov class in $L$ whenever $\Subset_{\mathscr{A}}$ also satisfies conditions (K3) and (K4). By Lemma 6.2, each Katětov class $\mathscr{A}$ in any $\mathscr{A}$-normal frame induces a Katětov relation $\Subset_{\mathscr{A}}$. The following proposition is obvious.

Proposition 6.3. Every sublattice $\mathscr{A}$ of $B(\mathcal{S}(L))$ is a Katětov class.

As a consequence, guiding classes $\mathscr{A}_{1}, \mathscr{A}_{3}, \mathscr{A}_{4}$ and $\mathscr{A}_{5}$, as well as their corresponding sets of complements $\mathscr{A}_{1}{ }^{\mathrm{c}}, \mathscr{A}_{3}{ }^{\mathrm{c}}, \mathscr{A}_{4}{ }^{\mathrm{c}}$ and $\mathscr{A}_{5}{ }^{\mathrm{c}}$, are Katětov classes in any frame. $\mathscr{A}_{2}$ and $\mathscr{A}_{2}{ }^{\mathrm{c}}$, despite the fact that they are not sublattices of $B(\mathcal{S}(L))$, also fit in this scheme as the following results show. 
Proposition 6.4. Let $\mathscr{A}$ be a class of complemented sublocales of L satisfying

$$
\left.\begin{array}{l}
\text { - } U_{1}, U_{2} \in \mathscr{A} \Rightarrow U_{1} \vee U_{2} \in \mathscr{A} \quad \text { (closed under binary joins) } \\
\text { - } U_{1}, U_{2} \in \mathscr{A}, U_{1} \wedge U_{2} \geq V \in \mathscr{A}^{\mathrm{c}} \Rightarrow \exists U \in \mathscr{A}: U_{1} \wedge U_{2} \geq U \geq V .
\end{array}\right\}
$$

Then:

(1) $\mathscr{A}$ is a Katětov class.

(2) If L is $\mathscr{A}^{\mathrm{c}}$-normal then $\mathscr{A}^{\mathrm{c}}$ is also a Katětov class.

Proof. (1) (K3): Let $S_{i} \leq U_{i}^{\prime} \leq U_{i} \leq T$ with $U_{i}^{\prime} \in \mathscr{A}^{\mathrm{c}}$ and $U_{i} \in \mathscr{A}(i=1,2)$. Then

$$
S_{1} \vee S_{2} \leq U_{1}^{\prime} \vee U_{2}^{\prime} \leq U_{1} \vee U_{2} \leq T,
$$

where $U_{1} \vee U_{2} \in \mathscr{A}$ (since $\mathscr{A}$ is closed under binary joins). In particular, applying $\left(*_{\wedge}\right)$ to the inequality $\left(U_{1}^{\prime}\right)^{\mathrm{c}} \wedge\left(U_{2}^{\prime}\right)^{\mathrm{c}} \geq\left(U_{1}\right)^{\mathrm{c}} \wedge\left(U_{2}\right)^{\mathrm{c}} \in \mathscr{A}^{\mathrm{c}}$, we get $U \in \mathscr{A}$ such that $U_{1}^{\prime} \vee U_{2}^{\prime} \leq U^{\mathrm{c}} \leq U_{1} \vee U_{2}$. Hence $S_{1} \vee S_{2} \leq U^{\mathrm{c}} \leq U_{1} \vee U_{2} \leq T$, i.e. $S_{1} \vee S_{2} \Subset_{\mathscr{A}} T$ as required.

(K4): Let $S \leq U_{i}^{\prime} \leq U_{i} \leq T_{i}$ with $U_{i}^{\prime} \in \mathscr{A}^{\mathrm{c}}$ and $U_{i} \in \mathscr{A}(i=1,2)$. Then

$$
S \leq U_{1}^{\prime} \wedge U_{2}^{\prime} \leq U_{1} \wedge U_{2} \leq T_{1} \wedge T_{2},
$$

where $U_{1}^{\prime} \wedge U_{2}^{\prime} \in \mathscr{A}^{\mathrm{c}}$ (since $\mathscr{A}^{\mathrm{c}}$ is closed under binary joins). In particular, applying $\left(*_{\wedge}\right)$ to the inequality $U_{1} \wedge U_{2} \geq U_{1}^{\prime} \wedge U_{2}^{\prime} \in \mathscr{A}^{\mathrm{c}}$, we get $U \in \mathscr{A}$ such that $U_{1}^{\prime} \wedge U_{2}^{\prime} \leq U \leq U_{1} \wedge U_{2}$. Hence $S \leq U_{1}^{\prime} \wedge U_{2}^{\prime} \leq U \leq T_{1} \wedge T_{2}$, i.e. $S \Subset_{\mathscr{A}} T_{1} \wedge T_{2}$ as required.

(2) (K3): Let $S_{i} \leq U_{i} \leq U_{i}^{\prime} \leq T$ with $U_{i} \in \mathscr{A}$ and $U_{i}^{\prime} \in \mathscr{A}^{\mathrm{c}}(i=1,2)$. Then $U_{i} \Subset_{\mathscr{A}^{\mathrm{c}}} U_{i}^{\prime}$. By Lemma 6.2, $\Subset_{\mathscr{A} c}$ is interpolative and so we have furthermore

$$
S_{i} \leq U_{i} \leq V_{i}^{\prime} \leq V_{i} \leq U_{i}^{\prime} \leq T
$$

for some $V_{i} \in \mathscr{A}$ and $V_{i}^{\prime} \in \mathscr{A}^{\mathrm{c}}$. Thus

$$
S_{1} \vee S_{2} \leq U_{1} \vee U_{2} \leq V_{1}^{\prime} \vee V_{2}^{\prime} \leq V_{1} \vee V_{2} \leq U_{1}^{\prime} \vee U_{2}^{\prime} \leq T
$$

where $U_{1} \vee U_{2}, V_{1} \vee V_{2} \in \mathscr{A}$ (since $\mathscr{A}$ is closed under binary joins). Then, applying (*^) to the inequality $\left(V_{1}^{\prime}\right)^{\mathrm{c}} \wedge\left(V_{2}^{\prime}\right)^{\mathrm{c}} \geq\left(V_{1}\right)^{\mathrm{c}} \wedge\left(V_{2}\right)^{\mathrm{c}} \in \mathscr{A}^{\mathrm{c}}$, we get $U \in \mathscr{A}$ such that $V_{1}^{\prime} \vee V_{2}^{\prime} \leq U^{\mathrm{c}} \leq V_{1} \vee V_{2}$. Hence $S_{1} \vee S_{2} \leq U_{1} \vee U_{2} \leq U^{\mathrm{c}} \leq T$, i.e. $S_{1} \vee S_{2} \Subset_{\mathscr{A}^{c}} T$ follows, as required.

(K4): Let $S \leq U_{i} \leq U_{i}^{\prime} \leq T_{i}$ with $U_{i} \in \mathscr{A}$ and $U_{i}^{\prime} \in \mathscr{A}^{\mathrm{c}}(i=1,2)$. Then $U_{i} \Subset_{\mathscr{A}^{c}} U_{i}^{\prime}$. By Lemma 6.2, $\Subset_{\mathscr{A} c}$ is interpolative and so we have furthermore

$$
S \leq U_{i} \leq V_{i}^{\prime} \leq V_{i} \leq U_{i}^{\prime} \leq T_{i}
$$

for some $V_{i} \in \mathscr{A}$ and $V_{i}^{\prime} \in \mathscr{A}^{\mathrm{c}}$. Thus

$$
S \leq U_{1} \wedge U_{2} \leq V_{1}^{\prime} \wedge V_{2}^{\prime} \leq V_{1} \wedge V_{2} \leq U_{1}^{\prime} \wedge U_{2}^{\prime} \leq T_{1} \wedge T_{2}
$$

where $U_{1}^{\prime} \wedge U_{2}^{\prime}, V_{1}^{\prime} \wedge V_{2}^{\prime} \in \mathscr{A}^{\mathrm{c}}$ (since $\mathscr{A}^{\mathrm{c}}$ is closed under binary meets). In particular, applying $\left(*_{\wedge}\right)$ to the inequality $V_{1} \wedge V_{2} \geq V_{1}^{\prime} \wedge V_{2}^{\prime} \in \mathscr{A}^{\mathrm{c}}$, we get $U \in \mathscr{A}$ such that $V_{1}^{\prime} \wedge V_{2}^{\prime} \leq U \leq V_{1} \wedge V_{2}$. Hence $S \leq U \leq U_{1}^{\prime} \wedge U_{2}^{\prime} \leq T_{1} \wedge T_{2}$, i.e. $S \Subset_{\mathscr{A}^{c}} T_{1} \wedge T_{2}$ as required.

The dual version of Proposition 6.4 follows immediately by complementation (since $\mathscr{A}$ satisfies $\left(*_{\vee}\right)$ if and only if $\mathscr{A}^{\mathrm{C}}$ satisfies $\left.\left(*_{\wedge}\right)\right)$ : 
Proposition 6.5. Let $\mathscr{A}$ be a class of complemented sublocales of L satisfying

$$
\left.\begin{array}{l}
\text { - } U_{1}, U_{2} \in \mathscr{A} \Rightarrow U_{1} \wedge U_{2} \in \mathscr{A} \quad \text { (closed under binary meets) } \\
\text { - } U_{1}, U_{2} \in \mathscr{A}, U_{1} \vee U_{2} \leq V \in \mathscr{A}^{\mathrm{c}} \Rightarrow \exists U \in \mathscr{A}: U_{1} \vee U_{2} \leq U \leq V .
\end{array}\right\}
$$

Then:

(1) $\mathscr{A}^{\mathrm{C}}$ is a Katětov class.

(2) If $L$ is $\mathscr{A}$-normal then $\mathscr{A}$ is also a Katětov class.

Note that Proposition 6.3 is an immediate consequence of Propositions 6.4 and 6.5 since $\mathscr{A}$ being closed under binary meets and joins implies both conditions $\left(*_{\wedge}\right)$ and $\left(*_{\vee}\right)$.

Corollary 6.6. $\mathscr{A}_{2}$ is a Katětov class in any mildly normal frame and $\mathscr{A}_{2}{ }^{\mathrm{c}}$ is a Katětov class in any frame.

Proof. It suffices to check that $\mathscr{A}_{2}$ satisfies condition $\left(*_{\mathrm{v}}\right) . \mathscr{A}_{2}$ is certainly closed under binary meets and, moreover, for any regular elements $a_{1}, a_{2}$ and $b$ in $L, \mathfrak{c}\left(a_{1}\right) \vee \mathfrak{c}\left(a_{2}\right) \leq \mathfrak{D}(b)$ implies $\mathfrak{c}\left(a_{1}\right) \vee \mathfrak{c}\left(a_{2}\right) \leq \mathfrak{c}\left(\left(a_{1} \vee a_{2}\right)^{* *}\right) \leq \mathfrak{v}\left(\left(a_{1} \vee a_{2}\right)^{*}\right) \leq \mathfrak{v}(b)$ and $\left(a_{1} \vee a_{2}\right)^{* *}$ is regular.

\section{Katětov-Tong-type insertion versus Stone-type insertion}

Finally, we come to the main result of the paper:

Theorem 7.1. [RELATIVE VERSION OF KaTĚTOV-TONG INSERTION THEOREM] Let $\mathscr{A} \subseteq B(\mathcal{S}(L))$ be a Katětov class. The following are equivalent:

(i) $L$ is $\mathscr{A}$-normal.

(ii) If $f \leq g$ are real functions on $L$ such that $f$ is upper and $g$ is lower $\mathscr{A}$-semicontinuous, then there exists an $\mathscr{A}$-continuous real function $h$ on $L$ such that $f \leq h \leq g$.

(iii) Every $S, T \in \mathscr{A}$ satisfying $S \vee T=1$ are completely $\mathscr{A}$-separated.

Proof. (i) $\Rightarrow$ (ii): Let $f \leq g$ be two real functions on $L$ such that $f$ is upper and $g$ is lower $\mathscr{A}$-semicontinuous and let $\Subset_{\mathscr{A}}$ be the Katětov relation defined in $\left(\Subset_{\mathscr{A}}\right)$. Further, let $a_{r}=f(-, r)^{*}$ and $b_{r}=g(r,-)$ for each $r \in \mathbb{Q}$. It follows from Remark 1.2(2) that $\left(a_{r} \mid r \in \mathbb{Q}\right)$ and $\left(b_{r} \mid r \in \mathbb{Q}\right)$ are scales generating $f$ and $g$, respectively. Hence, in particular,

$$
a_{q} \leq a_{p} \quad \text { and } \quad b_{q} \leq b_{p} \quad \text { whenever } p<q .
$$

On the other hand, let $p, r, s, q \in \mathbb{Q}$ such that $p<r<s<q$. Since $f$ is upper and $g$ is lower $\mathscr{A}$-semicontinuous, there exist $S_{s, q}, T_{p, r} \in \mathscr{A}$ such that

$$
f(-, s) \leq S_{s, q} \leq f(-, q) \quad \text { and } \quad g(r,-) \leq T_{p, r} \leq g(p,-) .
$$

Finally, since $f \leq g$, it follows from Lemma 1.3 that

$$
a_{q}=f(-, q)^{*} \leq S_{s, q}{ }^{\mathrm{c}} \leq f(-, s)^{*} \leq f(r,-) \leq g(r,-) \leq T_{p, r} \leq g(p,-)=b_{p},
$$

i.e. $a_{q} \Subset_{\mathscr{A}} b_{p}$.

We can now apply Lemma 6.1 with

$$
M \equiv \mathcal{S}(L), \quad \Subset \equiv \Subset_{\mathscr{A}}, \quad D \equiv \mathbb{Q} \quad \text { and } \quad \triangleleft \equiv<.
$$


Hence there exists a family $\left(c_{r} \mid r \in \mathbb{Q}\right)$ of sublocales of $L$ such that

$$
c_{q} \Subset_{\mathscr{A}} c_{p}, \quad a_{q} \Subset_{\mathscr{A}} c_{p} \quad \text { and } \quad c_{q} \Subset_{\mathscr{A}} b_{p} \quad \text { whenever } p<q .
$$

First note that $\bigvee_{p \in \mathbb{Q}} c_{p} \geq \bigvee_{p \in \mathbb{Q}} a_{p}=1$ and $\bigvee_{p \in \mathbb{Q}} c_{p}{ }^{*} \geq \bigvee_{p \in \mathbb{Q}} b_{p}{ }^{*}=1$. Also, if $p<q$ then $c_{q} \Subset_{\mathscr{A}} c_{p}$ and so there exists $U \in \mathscr{A}$ such that $c_{q} \leq U \leq c_{p}$. Since $U$ is complemented, it follows from Remark 1.2(1) that $\left(c_{r} \mid r \in \mathbb{Q}\right)$ satisfies (S1). Hence $\left(c_{r} \mid r \in \mathbb{Q}\right)$ is a scale and the generated function $h$ satisfies:

(1) $f \leq h, \quad$ (since $a_{q} \leq c_{p}$ whenever $p<q$, applying Lemma 1.3).

(2) $h \leq g, \quad$ (since $c_{q} \leq b_{p}$ whenever $p<q$, applying Lemma 1.3).

(3) $h$ is $\mathscr{A}$-continuous:

Let $p<r<q$, since $c_{q} \Subset_{\mathscr{A}} c_{p}$, it follows that there exist $T_{r, q}, T_{p, r} \in \mathscr{A}^{\mathrm{C}}$ and $S_{r, q}, S_{p, r} \in \mathscr{A}$ such that $c_{q} \leq T_{r, q} \leq S_{r, q} \leq c_{r}$ and $c_{r} \leq T_{p, r} \leq S_{p, r} \leq c_{p}$. Hence

$$
h(q,-)=\bigvee_{q^{\prime}>q} c_{q^{\prime}} \leq c_{q} \leq S_{r, q} \leq c_{r} \leq \bigvee_{p^{\prime}>p} c_{p^{\prime}}=h(p,-)
$$

and

$$
h(-, p)=\bigvee_{p^{\prime}<p} c_{p^{\prime}}{ }^{*} \leq c_{p}{ }^{*} \leq T_{p, r}{ }^{c} \leq c_{r}{ }^{*} \leq \bigvee_{q^{\prime}<q} c_{q^{\prime}}{ }^{*}=h(-, q)
$$

(ii) $\Rightarrow$ (iii): Let $S, T \in \mathscr{A}$ satisfying $S \vee T=1$. Then $\chi_{T}$ is upper $\mathscr{A}$-semicontinuous, $\chi_{S^{c}}$ is lower $\mathscr{A}$-semicontinuous and $\chi_{T} \leq \chi_{S^{c}}$. Hence, by hypothesis, there is an $\mathscr{A}$-continuous real function $h$ such that $\chi_{T} \leq h \leq \chi_{S^{c}}$. But this means that $h(-, 1) \leq \chi_{T}(-, 1)=T$ and $h(0,-) \leq \chi_{S^{c}}(0,-)=S$ and thus $S$ and $T$ are completely $\mathscr{A}$-separated.

(iii) $\Rightarrow$ (i): Let $A, B \in \mathscr{A}$ with $A \vee B=1$. By hypothesis, there is an $\mathscr{A}$-continuous $f$ such that $f(0,-) \leq A$ and $f(-, 1) \leq B$. Consider $U, V \in \mathscr{A}$ such that

$$
f\left(-, \frac{1}{4}\right) \leq U \leq f\left(-, \frac{1}{2}\right) \quad \text { and } \quad f\left(\frac{3}{4},-\right) \leq V \leq f\left(\frac{1}{2},-\right) .
$$

Clearly, $U \wedge V=0$ and $A \vee U=1=B \vee V$.

Now the dual result for extremal $\mathscr{A}$-disconnectedness follows immediately by complementation:

Corollary 7.2. Let $\mathscr{A} \subseteq B(\mathcal{S}(L))$ be such that $\mathscr{A}^{\mathrm{c}}$ is a Katětov class. The following are equivalent:

(i) $L$ is $\mathscr{A}$-disconnected.

(ii) If $g \leq f$ are real functions on $L$ such that $f$ is upper and $g$ is lower $\mathscr{A}$-semicontinuous, then there exists an $\mathscr{A}$-continuous real function $h$ on $L$ such that $g \leq h \leq f$.

(iii) Every $S, T \in \mathscr{A}$ satisfying $S \wedge T=0$ are completely $\mathscr{A}$-separated.

Notes 7.3. (1) When considering the excellent survey in Lane's paper [35], we observe that Theorem 7.1 and Corollary 7.2 cover all the cases mentioned there where the classes of upper and lower semicontinuous functions in question are dual to each other. More specifically:

It is clear that Theorem 7.1 applied to $\mathscr{A}=\mathscr{A}_{1}$ provides the pointfree version of the KatětovTong insertion theorem [20, 17] (as well as Urysohn's Lemma) while its corollary produces the pointfree counterpart of Stone insertion theorem [15]. 
Applying Theorem 7.1 to the case $\mathscr{A}=\mathscr{A}_{2}$ yields a new result for mildly normal frames that extends the classical result of [34] (cf. [35]) for mildly normal spaces; on the other hand, Corollary 7.2 yields the pointfree counterpart (for extremally disconnected frames) of a classical result of Lane [35].

The case $\mathscr{A}=\mathscr{A}_{3}$ also produces new results for frames: the 'normality' assertion extends a classical result of Blatter and Seever [8] (cf. [35]) and the 'extremal disconnectedness' dual extends a classical result of Seever [41] (cf. [35]).

Finally, we mention that the case $\mathscr{A}=\mathscr{A}_{5}$ also produces new results for frames and that we were not able to find their classical counterparts in the literature.

(2) With Corollary 7.2 we achieved our main goal: to get the results in the extremal disconnectedness side of the parallel for free. Compare this with the (classical and pointfree) proofs so far where the treatment in the extremal disconnectedness case requires extra tools such as the lower and upper regularizations.

(3) Of course, applying Theorem 7.1 and Corollary 7.2 to the case $L=O X$ for a topological space $(X, O X)$ gives the classical results mentioned above in (1). (Recall Remark 4.2).

\section{Tietze-type extension for $\mathscr{A}$-continuous functions}

In this section we study Tietze-type extension results for $\mathscr{A}$-continuous real functions.

Let $S$ be a sublocale of $L . S$ is itself a frame with the same meets as in $L$, and since the Heyting operation $\rightarrow$ depends on the meet structure only, with the same Heyting operation. (This implies, in particular, that any sublocale of $S$ is also a sublocale of $L$ and that for any $S, T \in \mathcal{S}(L)$, if $S \subseteq T$ then $S$ is also a sublocale of $T$.) However the joins in $S$ and $L$ will not necessarily coincide:

$$
\bigvee^{S} A=\bigwedge\{s \in S \mid s \geq \bigvee A\} \geq \bigvee A \text {. }
$$

It follows that $1_{S}=1$ but in general $0_{S}=\bigwedge S$.

Further, there is the frame surjection $c_{S}: L \rightarrow S$ given by

$$
c_{S}(x)=\bigwedge\{s \in S \mid s \geq x\}
$$

for all $x \in L$. The following is well known (denoting the closed and open sublocales of $S$ by $\mathfrak{c}^{S}(a)$ and $\mathfrak{o}^{S}(a)$, respectively) (see [11, Prop. 2.3]):

(1) For every $a \in L, \mathfrak{c}(a) \vee S$ (resp. $\mathfrak{D}(a) \vee S)$ is the closed (resp. open) sublocale $\mathfrak{c}^{S}\left(c_{S}(a)\right)$ (resp. $\left.\mathfrak{o}^{S}\left(c_{S}(a)\right)\right)$ of $S$.

(2) If $T$ is a closed (resp. open) sublocale of $S$ then $T=\mathfrak{c}(a) \vee S$ (resp. $T=\mathfrak{v}(a) \vee S$ ) for some $a \in L$.

Now, let us look to the frame $\mathcal{S}(S)$. As mentioned above, $\mathcal{S}(S) \subseteq \mathcal{S}(L)$. The joins in $\mathcal{S}(S)$ are given by intersection so they coincide with the joins in $\mathcal{S}(L)$. On the other hand, for any $R_{i} \in \mathcal{S}(S)$ their meet in $\mathcal{S}(S)$ is given by the formula

$$
\left\{\stackrel{S}{\wedge} A \mid A \subseteq \bigcup_{i} R_{i}\right\}=\left\{\bigwedge^{L} A \mid A \subseteq \bigcup_{i} R_{i}\right\},
$$

that is, it coincides with their meet in $\mathcal{S}(L)$. (This means that $\mathcal{S}(S)$ is not a subframe of $\mathcal{S}(L)$ only because they have different zeros.) The following is also well known and easy to prove. 
Lemma 8.1. Let $S$ be a sublocale of $L$. The map $\varphi_{S}: \mathcal{S}(L) \rightarrow \mathcal{S}(S)$ given by $\varphi_{S}(T)=T \vee S=$ $T \cap S$ is an onto frame homomorphism.

This means that $\mathcal{S}(S)$ is a sublocale of $\mathcal{S}(L)$ and allows us to introduce the following definition.

Definition 8.2. Let $S$ be a sublocale of $L$ and $\mathscr{A} \subseteq B(\mathcal{S}(L))$. We say that an $f: \mathbb{I}(\mathbb{R}) \rightarrow \mathcal{S}(S)$ in $\mathrm{F}(S)$ has an $\mathscr{A}$-continuous extension to $L$ if there exists an $\mathscr{A}$-continuous $\tilde{f}: \mathfrak{L}(\mathbb{R}) \rightarrow \mathcal{S}(L)$ such that the diagram

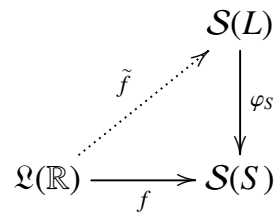

commutes. The extension $\tilde{f}$ is called an $\mathscr{A}$-continuous extension of $f$ to $L$.

Remarks 8.3. (1) Note that $\varphi_{S}$ is precisely the quotient $c_{\mathcal{S}(S)}: \mathcal{S}(L) \rightarrow \mathcal{S}(S)$ from (8.1) since

$$
c_{\mathcal{S}(S)}(T)=\bigwedge\{R \in \mathcal{S}(S) \mid T \leq R\}=\left\{\bigwedge A \mid A \subseteq \bigcup_{R \in \mathcal{S}(S), R \subseteq T} R\right\}=T \cap S
$$

(in the last equality, ' $\subseteq$ ' is obvious; conversely, if $x \in T \cap S$ then, since $T \cap S \in \mathcal{S}(S), A=\{x\} \subseteq$ $\left.\bigcup_{R \in \mathcal{S}(S), R \subseteq T} R\right)$.

(2) For $f \in \mathrm{C}(S)$ and $\mathscr{A}=\mathfrak{c}(L)$ this notion coincides with the previous notion in the Appendix to [17] because $\varphi_{S}=c_{\mathcal{S}(S)}$, by (1), and by (S1) the restriction of $c_{\mathcal{S}(S)}$ to $\mathfrak{c}(L)$ is precisely the frame homomorphism $c_{\mathfrak{c}(S)}: \mathfrak{c}(L) \rightarrow \mathfrak{c}(S)$ used in [17].

Definitions 8.4. We say that a Katětov class $\mathscr{A}$ of a frame $L$ is a Tietze class whenever

(T1) $\mathscr{A}$ is closed under finite meets, and

(T2) $\mathscr{A}$ is closed under countable joins.

Given a Tietze class $\mathscr{A}$ and a sublocale $S$ of $L$, let

$$
\mathscr{A}_{S}=\{S \vee A \mid A \in \mathscr{A}\} \text {. }
$$

Of course, $\mathscr{A}_{S} \subseteq \mathscr{A}$ whenever $S \in \mathscr{A}$. We say that a sublocale $S$ is $C_{\mathscr{A}}$-embedded if every $\mathscr{A}_{S}$ continuous $f \in \mathrm{F}(S)$ has an $\mathscr{A}$-continuous extension to $L$. Further, $S$ is said to be $C_{\mathscr{A}}^{*}$-embedded if every bounded $\mathscr{A}_{S}$-continuous $f \in \mathrm{F}(S)$ has an $\mathscr{A}$-continuous extension to $L$ (recall that an $f \in \mathrm{F}(S)$ is bounded if there exist rationals $p, q$ such that $\boldsymbol{p} \leq f \leq \boldsymbol{q}$, i.e. $f(-, p)=f(q,-)=$ $\left.0_{\mathcal{S}(S)}=S\right)$.

Examples 8.5. Each of the guiding examples $\mathscr{A}_{1}, \mathscr{A}_{3}$ and $\mathscr{A}_{5}$ is a Tietze class. Note that condition (T2) is not self-dual and is responsible for the fact that $\mathscr{A}_{1}^{\mathrm{c}}=\{\mathrm{p}(a) \mid a \in L\}$ is not a Tietze class.

Theorem 8.6. [Relative version of Tietze's extension theorem] Let $\mathscr{A}$ be a Tietze class of L. The following are equivalent:

(i) Lis $\mathscr{A}$-normal.

(ii) Every $S \in \mathscr{A}$ is $C_{\mathscr{A}}^{*}$-embedded in L. 
Proof. (i) $\Rightarrow$ (ii): Let $S \in \mathscr{A}$ and let $f: \mathfrak{L}(\mathbb{R}) \rightarrow \mathcal{S}(S)$ be a bounded $\mathscr{A}_{S}$-continuous map (we can assume w.l.o.g. that $\mathbf{0} \leq f \leq \mathbf{1}$, i.e. $\left.f(-, 0)=f(1,-)=0_{\mathcal{S}(S)}=S\right)$. Then, for each $p<q$ there are $U_{p, q}, V_{p, q} \in \mathscr{A}_{S}$ such that $f(-, p) \leq U_{p, q} \leq f(-, q)$ and $f(q,-) \leq V_{p, q} \leq f(p,-)$. In particular, $f(-, q)=\bigvee_{p<q} U_{p, q}$ and $f(p,-)=\bigvee_{q>p} V_{p, q}$ and thus, by (T2), $f(-, q), f(p,-) \in \mathscr{A}$. Moreover, since $f(p,-) \vee f(-, q)=1_{\mathcal{S}(S)}=1_{\mathcal{S}(L)}=\{1\}$ for every $p<q$, then

$$
\bigvee_{r<q} U_{r, q} \vee \bigvee_{s>p} V_{p, s}=\{1\} \quad \text { for evey } p<q
$$

from which it follows that

$$
\bigwedge_{r<q} U_{r, q}{ }^{c}=\left(\bigvee_{r<q} U_{r, q}\right)^{*} \leq \bigvee_{s>p} V_{p, s} \quad \text { for evey } p<q .
$$

Define an antitone $\left(S_{r} \mid r \in \mathbb{Q}\right) \subseteq \mathcal{S}(L)$ as follows:

$$
S_{r}= \begin{cases}1_{\mathcal{S}(L)}=\{1\} & \text { if } r<0 \\ f(r,-) & \text { if } 0 \leq r<1 \\ 0_{\mathcal{S}(L)}=L & \text { if } r \geq 1 .\end{cases}
$$

This is a scale that generates a $g_{2} \in \mathrm{F}(L)$, given by

$$
g_{2}(p,-)= \begin{cases}\{1\} & \text { if } p<0 \\ \bigvee_{q>p} V_{p, q} & \text { if } 0 \leq p<1 \\ L & \text { if } p \geq 1\end{cases}
$$

and

$$
g_{2}(-, q)= \begin{cases}\{1\} & \text { if } q>1 \\ \bigvee_{r<q} f(r,-)^{*}=\bigvee_{r<q} \bigwedge_{t>r} V_{r, t}{ }^{c} & \text { if } 0<q \leq 1 \\ L & \text { if } q \leq 0 .\end{cases}
$$

Clearly, $g_{2}$ is a lower $\mathscr{A}$-continuous map. Similarly,

$$
g_{1}(-, q)= \begin{cases}\{1\} & \text { if } q>1 \\ \bigvee_{p<q} U_{p, q} & \text { if } 0<q \leq 1 \\ L & \text { if } q \leq 0\end{cases}
$$

and

$$
g_{1}(p,-)= \begin{cases}\{1\} & \text { if } p<0 \\ \bigvee_{s>p} f(-, s)^{*}=\bigvee_{s>p} \wedge_{t<s} U_{t, s}{ }^{c} & \text { if } 0 \leq p<1 \\ L & \text { if } p \geq 1\end{cases}
$$

define an upper $\mathscr{A}$-continuous map and by (8.2) we have $g_{1} \leq g_{2}$.

It then follows from Theorem 7.1 that there exists an $\mathscr{A}$-continuous $h \in \mathrm{F}(L)$ such that $g_{1} \leq h \leq g_{2}$. This is the desired $\mathscr{A}$-continuous extension of the given $f$. Indeed:

- For every $p<0, h(p,-) \geq g_{1}(p,-)=\{1\}$, so $\left(\varphi_{S} \cdot h\right)(p,-)=f(p,-)$.

- For every $p \geq 1, h(p,-) \leq g_{2}(p,-)=L$, so $\left(\varphi_{S} \cdot h\right)(p,-)=S=f(p,-)$.

- For every $q>1, h(-, q) \geq g_{2}(q,-)=1$, so $\left(\varphi_{S} \cdot h\right)(-, q)=f(-, q)$. 
- For every $q \leq 0, h(-, q) \leq g_{1}(-, q)=0$, so $\left(\varphi_{S} \cdot h\right)(-, q)=S=f(-, q)$.

- For every $0 \leq p<1$ and for each $r \in \mathbb{Q}$ such that $p<r<1$ we have

$$
\begin{aligned}
f(r,-) & =f(r,-) \wedge(h(p,-) \vee h(-, r))=(f(r,-) \wedge h(p,-)) \vee(f(r,-) \wedge h(-, r)) \\
& \leq h(p,-) \vee\left(f(r,-) \wedge g_{1}(-, r)\right)=h(p,-) \vee(f(r,-) \wedge f(-, r)) \\
& =h(p,-) \vee 0_{\mathcal{S}(S)}=h(p,-) \vee S .
\end{aligned}
$$

Hence $f(p,-)=\left(\bigvee_{p<r<1} f(r,-)\right) \leq h(p,-) \vee S \leq g_{2}(p,-) \vee S=f(p,-)$ and so $\left(\varphi_{S} \cdot h\right)(p,-)=$ $h(p,-) \vee S=f(p,-)$.

- For every $0<q \leq 1$ we can prove in a similar way that $\left(\varphi_{S} \cdot h\right)(-, q)=f(-, q)$.

(ii) $\Rightarrow$ (i): Conversely, suppose $A \vee B=1_{\mathcal{S}(L)}=\{1\}$ with $A, B \in \mathscr{A}$ and consider the sublocale $S=A \wedge B$ which by (T1) also belongs to $\mathscr{A}$. Define $f: \mathfrak{L}(\mathbb{R}) \rightarrow \mathcal{S}(S)$ by

$$
f(p,-)=\left\{\begin{array}{ll}
1_{\mathcal{S}(S)}=\{1\} & \text { if } p<0 \\
A & \text { if } 0 \leq p<1 \\
0_{\mathcal{S}(S)}=S & \text { if } p \geq 1
\end{array} \quad \text { and } \quad f(-, q)= \begin{cases}1_{\mathcal{S}(S)}=\{1\} & \text { if } q>1 \\
B & \text { if } 0<q \leq 1 \\
0_{\mathcal{S}(S)}=S & \text { if } q \leq 0 .\end{cases}\right.
$$

This is a frame homomorphism. Since $A, B, S,\{1\} \in \mathscr{A}_{S}$, this is moreover a bounded $\mathscr{A}_{S}$-continuous map. Then it has an $\mathscr{A}$-continuous extension $\tilde{f}: \mathfrak{L}(\mathbb{R}) \rightarrow \mathcal{S}(L)$. We have

$$
\tilde{f}(0,-) \leq \varphi_{S}(\tilde{f}(0,-))=f(0,-)=A \quad \text { and } \quad \tilde{f}(-, 1) \leq \varphi_{S}(\tilde{f}(-, 1))=f(-, 1)=B
$$

and so $A$ and $B$ are completely $\mathscr{A}$-separated in $L$. Therefore $L$ is $\mathscr{A}$-normal by Theorem 7.1.

Once again the dual result for extremal $\mathscr{A}$-disconnectedness follows immediately by complementation:

Corollary 8.7. Let $\mathscr{A} \subseteq B(\mathcal{S}(L))$ be such that $\mathscr{A}^{\mathrm{C}}$ is a Tietze class of $L$. The following are equivalent:

(i) L is $\mathscr{A}$-disconnected.

(ii) Every $S \in \mathscr{A}^{\mathrm{C}}$ is $C_{\mathscr{A}^{-}}^{*}$-embedded in $L$.

\section{Images of $\mathscr{A}$-normal and $\mathscr{A}$-disconnected locales}

In this final section we show how our general approach in Section 4 also makes possible to extend to locales the Hausdorff mapping invariance type theorems (Table 1) and to prove their relative versions with a single proof. For that we need first to recall a few facts about localic maps from [39].

A map $f: L \rightarrow M$ between locales $L$ and $M$ is a localic map if it has a left adjoint $f^{*}: M \rightarrow L$ such that $f^{*}(1)=1$ and $f^{*}(a \wedge b)=f^{*}(a) \wedge f^{*}(b)$. Then:

(1) $f$ preserves arbitrary meets (in particular, $f(1)=1$ ).

(2) If $f(a)=1$ then $a=1$.

For each $S \in \mathcal{S}(L)$, the image of $S, f[S]$, is a sublocale of $M$. From [39] we know that

$$
f[-]: \mathcal{S}(L) \rightarrow \mathcal{S}(M)
$$

is a localic map. The localic map $f$ is closed if the image of each closed sublocale of $L$ is closed. In that case, $f[\mathfrak{c}(a)]=\mathfrak{c}(f(a))$ for each $a \in L$. Moreover: 
(1) $f[-]$ preserves arbitrary meets.

(2) $f\left[0_{\mathcal{S}(M)}\right]=0_{\mathcal{S}(M)}$ iff $f$ is surjective.

On the other hand, the preimage $f_{-1}[T]$ of a sublocale $T$ of $M$ is defined as

$$
f_{-1}[T]=\bigwedge\left\{S \in \mathcal{S}(L) \mid S \geq f^{-1}[T]\right\},
$$

where $f^{-1}[T]$ denotes the set-theoretical preimage of $T$ (which is only closed under meets, but not necessarily a sublocale of $L$ ). The function

$$
f_{-1}[-]: \mathcal{S}(M) \rightarrow \mathcal{S}(L)
$$

is left adjoint to $f[-]$, that is,

$$
f_{-1}[T] \leq S \text { iff } T \leq f[S] .
$$

The preimage of a closed (resp. open) sublocale under a localic map is closed (resp. open). Specifically,

$$
f_{-1}[\mathfrak{c}(a)]=\mathfrak{c}\left(f^{*}(a)\right) \text { and } f_{-1}[\mathfrak{p}(a)]=\mathfrak{v}\left(f^{*}(a)\right) \text { for each } a \in L .
$$

Moreover:

(1) $f_{-1}[-]$ preserves all joins and all finite meets.

(2) $f_{-1}[-]$ preserves complements.

Now we require some terminology. Let $(\mathscr{A})_{L}\left(\right.$ resp. $\left.(\mathscr{A})_{M}\right)$ denote a subset of the Boolean part of $\mathcal{S}(L)$ (resp. $\mathcal{S}(M))$. We say that a localic map $f: L \rightarrow M$ is $(\mathscr{A})_{L}(\mathscr{A})_{M}$-preserving if $f[-]$ maps elements of $(\mathscr{A})_{L}$ into $(\mathscr{A})_{M}$.

Examples 9.1. (1) $\left(\mathscr{A}_{1}\right)_{L}\left(\mathscr{A}_{1}\right)_{M}$-preserving localic maps are precisely the closed ones while $\left(\mathscr{A}_{1}^{\mathrm{c}}\right)_{L}\left(\mathscr{A}_{1}^{\mathrm{c}}\right)_{M}$-preserving localic maps are the open ones. Moreover, by $(9.1), f_{-1}[-]$ assigns elements of $\left(\mathscr{A}_{1}\right)_{M}$ into $\left(\mathscr{A}_{1}\right)_{L}$ and elements of $\left(\mathscr{A}_{1}^{\mathrm{c}}\right)_{M}$ into $\left(\mathscr{A}_{1}^{\mathrm{c}}\right)_{L}$.

(2) Regarding $\left(\mathscr{A}_{2}\right)_{L}\left(\mathscr{A}_{2}\right)_{M}$-preserving localic maps they are precisely the localic maps $f: L \rightarrow$ $M$ such that for each $a \in L$ there is some $b \in M$ satisfying $f\left[\mathfrak{c}\left(a^{*}\right)\right]=\mathfrak{c}\left(b^{*}\right)$, that is, $f\left[\uparrow a^{*}\right]=\uparrow b^{*}$. We can say more:

Proposition. For a localic map $f: L \rightarrow M$ the following are equivalent:

(i) $f$ is $\left(\mathscr{A}_{2}\right)_{L}\left(\mathscr{A}_{2}\right)_{M}$-preserving.

(ii) For each $a \in L, f\left[\uparrow a^{*}\right]=\uparrow f\left(a^{*}\right)$ and $f\left(a^{*}\right)$ is regular.

(iii) For each $a \in L$ and $b \in M, f\left(a^{*} \vee f^{*}(b)\right)=f\left(a^{*}\right) \vee b$ and $f\left(a^{*}\right)$ is regular.

Proof. (i) $\Rightarrow$ (ii): $f\left[\uparrow a^{*}\right]=\uparrow b^{*}$ for some $b$ and since $f\left(a^{*}\right)$ is obviously the minimal element in $f\left[\uparrow a^{*}\right], b^{*}=f\left(a^{*}\right)$ and $f\left(a^{*}\right)$ is regular.

(ii) $\Rightarrow$ (iii): We have always $f\left(a^{*} \vee f^{*}(b)\right) \geq f\left(a^{*}\right) \vee f f^{*}(b) \geq f\left(a^{*}\right) \vee b$. Moreover, $f\left(a^{*}\right) \vee b=f(x)$ for some $x \geq a^{*}$. As $f(x) \geq b$, we have $x \geq a^{*} \vee f^{*}(b)$, and $f\left(a^{*} \vee f^{*}(b)\right) \leq f(x)=f\left(a^{*}\right) \vee b$.

(iii) $\Rightarrow$ (i): If $x \geq f\left(a^{*}\right)$ then $x=f\left(a^{*}\right) \vee x=f\left(a^{*} \vee f^{*}(x)\right) \in f\left[\uparrow a^{*}\right]$. Hence $f\left[\uparrow a^{*}\right]=\uparrow f\left(a^{*}\right)$ and we have (ii). The fact that (ii) implies (i) is obvious. 
For instance, any localic closed map that preserves regular elements is $\left(\mathscr{A}_{2}\right)_{L}\left(\mathscr{A}_{2}\right)_{M}$-preserving. Note that by $(9.1)$, for any $f, f_{-1}[-]$ maps elements of $\left(\mathscr{A}_{2}\right)_{M}$ into $\left(\mathscr{A}_{2}\right)_{L}$ iff the left adjoint $f^{*}$ preserves regular elements (these are the frame homomorphisms classified as of type $\mathbf{E}$ in ([7], Theorem 3.5)). This is true, in particular, when $h=f^{*}$ is nearly open, that is, such that $h\left(a^{*}\right)=$ $h(a)^{*}$ (precisely the frame homomorphisms in class $\mathbf{A}$ of [7]). Moreover, for any such nearly open $h, f$ preserves regular elements; in fact, $h f\left(a^{*}\right) \leq a^{*}$ implies $h\left(f\left(a^{*}\right)^{* *}\right)=\left(h f\left(a^{*}\right)\right)^{* *} \leq a^{* * *}=a^{*}$, therefore, by adjunction, $f\left(a^{*}\right)^{* *} \leq f\left(a^{*}\right)$ and thus $f\left(a^{*}\right)^{* *}=f\left(a^{*}\right)$.

(3) Regarding $\left(\mathscr{A}_{3}\right)_{L}\left(\mathscr{A}_{3}\right)_{M}$-preserving localic maps we have a similar result:

Proposition. For a localic map $f: L \rightarrow M$ the following are equivalent:

(i) $f$ is $\left(\mathscr{A}_{3}\right)_{L}\left(\mathscr{A}_{3}\right)_{M}$-preserving.

(ii) For each $a \in \operatorname{Coz} L, f[\uparrow a]=\uparrow f(a)$ and $f(a) \in \operatorname{Coz} M$.

(iii) For each $a \in \operatorname{Coz} L$ and $b \in M, f\left(a \vee f^{*}(b)\right)=f(a) \vee b$ and $f(a) \in \operatorname{Coz} M$.

We note in addition that $f_{-1}[-]$ maps elements of $\left(\mathscr{A}_{3}\right)_{M}$ into $\left(\mathscr{A}_{3}\right)_{L}$ iff the frame homomorphism $f^{*}$ preserves cozero elements (which is always the case for any frame homomorphism).

The pointfree counterpart of the Hausdorff mapping invariance theorem is now a particular case of the following general result.

Theorem 9.2. [ReLATIVE Version of Hausdorff Mapping INVARiance Theorem]

Let $f: L \rightarrow M$ be an $(\mathscr{A})_{L}(\mathscr{A})_{M}$-preserving localic map such that $f_{-1}[-]$ maps elements of $(\mathscr{A})_{M}$ into $(\mathscr{A})_{L}$. If $L$ is $(\mathscr{A})_{L}$-normal and $f$ is a surjection then $M$ is $(\mathscr{A})_{M}$-normal.

Proof. Let $A$ and $B$ be elements of $(\mathscr{A})_{M}$ such that $A \vee B=1_{\mathcal{S}(M)}$. Since $f_{-1}[-]$ maps elements of $(\mathscr{A})_{M}$ into $(\mathscr{A})_{L}$ we have $f_{-1}[A], f_{-1}[B] \in(\mathscr{A})_{L}$ and since $f_{-1}[-]$ preserves joins, we have

$$
f_{-1}[A] \vee f_{-1}[B]=f_{-1}[A \vee B]=f_{-1}\left[1_{\mathcal{S}(M)}\right]=1_{\mathcal{S}(L)} .
$$

Therefore, applying the $(\mathscr{A})_{L}$-normality of $L$, we get sublocales $U_{0}$ and $V_{0}$ in $(\mathscr{A})_{L}$ such that $U_{0} \wedge V_{0}=0_{\mathcal{S}(L)}, f_{-1}[A] \vee U_{0}=1_{\mathcal{S}(L)}=f_{-1}[B] \vee V_{0}$. Since $f_{-1}[-]$ preserves complements we have $f_{-1}\left[A^{\mathrm{C}}\right]=f_{-1}[A]^{\mathrm{C}} \leq U_{0}$ and $f_{-1}\left[B^{\mathrm{C}}\right]=f_{-1}[B]^{\mathrm{C}} \leq V_{0}$. Let

$$
U=f\left[U_{0}\right] \in(\mathscr{A})_{M} \text { and } V=f\left[V_{0}\right] \in(\mathscr{A})_{M}
$$

(using the fact that $f$ is $(\mathscr{A})_{L}(\mathscr{A})_{M}$-preserving). Since $f$ is surjective and $f[-]$ preserves meets we have

$$
U \wedge V=f\left[U_{0}\right] \wedge f\left[V_{0}\right]=f\left[U_{0} \wedge V_{0}\right]=f\left[0_{\mathcal{S}(L)}\right]=0_{\mathcal{S}(M)} .
$$

On the other hand, $U \geq f f_{-1}\left[A^{\mathrm{c}}\right] \geq A^{\mathrm{c}}$ and similarly $V \geq f f_{-1}\left[B^{\mathrm{c}}\right] \geq B^{\mathrm{c}}$. Hence

$$
A \vee U=1_{\mathcal{S}(M)}=B \vee V .
$$

Again, by complementation, one gets immediately the following (observe that, since the preimage function preserves complements, saying that $f_{-1}[-]$ maps elements of $(\mathscr{A})_{M}^{\mathrm{c}}$ into $(\mathscr{A})_{L}^{\mathrm{c}}$ is the same as saying that $f_{-1}[-]$ maps elements of $(\mathscr{A})_{M}$ into $\left.(\mathscr{A})_{L}\right)$ :

Corollary 9.3. Let $f: L \rightarrow M$ be an $(\mathscr{A})_{L}^{\mathrm{c}}(\mathscr{A})_{M}^{\mathrm{c}}$-preserving localic map such that $f_{-1}[-]$ maps elements of $(\mathscr{A})_{M}$ into $(\mathscr{A})_{L}$. If $L$ is $(\mathscr{A})_{L}$-disconnected and $f$ is a surjection then $M$ is $(\mathscr{A})_{M-}$ disconnected. 
The particular case $\mathscr{A}=\mathscr{A}_{1}$ provides the following result, which completes Table 2 at the Introduction.

Corollary 9.4. Let $f: L \rightarrow M$ be a surjective localic map.

(1) If $f$ is closed and $L$ is normal then so is $M$.

(2) If $f$ is open and $L$ is extremally disconnected then so is $M$.

\section{References}

[1] R. N. Ball and J. Walters-Wayland, $C$ - and $C^{*}$-quotients in pointfree topology, Dissertationes Math. (Rozprawy Mat.) 412, 2002.

[2] B. Banaschewski, $\sigma$-frames, unpublished manuscript, 1980.

[3] B. Banaschewski, The real numbers in pointfree topology, Textos de Matemática, Vol. 12, University of Coimbra, 1997.

[4] B. Banaschewski, A new aspect of the cozero lattice in pointfree topology, Topology Appl. 156 (2009) $2028-2038$.

[5] B. Banaschewski and C. Gilmour, Pseudocompactness and the cozero part of a frame, Comment. Math. Univ. Carolin. 37 (1996) 577-587.

[6] B. Banaschewski, J. Gutiérrez García and J. Picado, Extended real functions in pointfree topology, J. Pure Appl. Algebra 216 (2012) 905-922.

[7] B. Banaschewski and A. Pultr, Variants of openness, Appl. Categ. Structures 2 (1994) 331-350.

[8] J. Blatter and G. L. Seever, Interposition of semicontinuous functions by continuous functions, Analyse Fonctionelle et Applications (Comptes Rendus du Colloque d'Analyse, Rio de Janeiro, 1972), pp. 27-51, Hermann, Paris, 1975.

[9] G. Buskes and A. van Rooij, Topological Spaces: From Distance to Neighborhood, Springer, New York, 1997.

[10] R. P. Dilworth, The normal completion of the lattice of continuous functions, Trans. Amer. Math. Soc. 68 (1950) 427-438.

[11] M. J. Ferreira, J. Gutiérrez García and J. Picado, Completely normal frames and real-valued functions, Topology Appl. 156 (2009) 2932-2941.

[12] L. Gillman and M. Jerison, Rings of continuous functions, Van Nostrand, 1960.

[13] J. Gutiérrez García and T. Kubiak, Sandwich-type characterizations of completely regular spaces, Appl. Gen. Topol. 8 (2007) 239-242.

[14] J. Gutiérrez García and T. Kubiak, General insertion and extension theorems for localic real functions, J. Pure Appl. Algebra 215 (2011) 1198-1204.

[15] J. Gutiérrez García, T. Kubiak and J. Picado, Lower and upper regularizations of frame semicontinuous real functions, Algebra Universalis 60 (2009) 169-84.

[16] J. Gutiérrez García, T. Kubiak and J. Picado, Pointfree forms of Dowker's and Michael's insertion theorems, $J$. Pure Appl. Algebra 213 (2009) 98-108.

[17] J. Gutiérrez García, T. Kubiak and J. Picado, Localic real-valued functions: a general setting, J. Pure Appl. Algebra 213 (2009) 1064-1074.

[18] J. Gutiérrez García, T. Kubiak and J. Picado, Perfectness in locales, in preparation.

[19] J. Gutiérrez García, T. Kubiak and M. A. de Prada Vicente, Insertion of lattice-valued and hedgehog-valued functions, Topology Appl. 153 (2006) 1458-1475.

[20] J. Gutiérrez García and J. Picado, On the algebraic representation of semicontinuity, J. Pure Appl. Algebra 210 (2007) 299-306.

[21] J. Gutiérrez García and J. Picado, Rings of real functions in pointfree topology, Topology Appl. 158 (2011) $2264-$ 2278.

[22] J. Gutiérrez García and J. Picado, Insertion and extension results for point-free complete regularity, Bull. Belg. Math. Soc. Simon Stevin, to appear.

[23] F. Hausdorff, Gestufte Räume, Fund. Math. 25 (1935) 486-502.

[24] J. Isbell, First steps in descriptive theory of locales, Trans. Amer. Math. Soc. 327 (1991) 353-371.

[25] E. P. de Jager and H.-P. A. Künzi, Permutable pairs of quasi-uniformities, Topology Appl. 158 (2011) 930-938.

[26] P. B. Johnson, $\kappa$-Lindelöf locales and their spatial parts, Cah. Topol. Géom. Différ. Catég. 32 (1991) $297-313$.

[27] P. T. Johnstone, Stone spaces, Cambridge University Press, Cambridge, 1982.

[28] M. Katětov, On real valued functions in topological spaces, Fund. Math. 38 (1951) 85-91; Fund. Math. 40 (1953) 139-142 (Correction).

[29] W. Kotzé and T. Kubiak, Insertion of a measurable function, J. Austral. Math. Soc. Ser. A 57 (1994) $295-304$.

[30] T. Kubiak, On Fuzzy Topologies, Ph. D. Thesis, UAM, Poznań, 1985. 
[31] T. Kubiak, On extremally disconnected subspaces, Fasc. Math. 19 (1990) 143-145.

[32] T. Kubiak, Separation axioms: extension of mappings and embedding of spaces, in: Mathematics of Fuzzy Sets: Logic, Topology and Measure Theory, The Handbooks of Fuzzy Sets Series, Vol.3 (ed. by U. Höhle and S. E. Rodabaugh), pp. 433-479, Kluwer, Dordrecht, 1999.

[33] T. Kubiak, Second open question, in: Applications of category theory to fuzzy subsets (ed. by S. E. Rodabaugh, E. P. Klement and U. Höhle), pp. 349, Kluwer, Dordrecht, 1992.

[34] E. P. Lane, A sufficient condition for the insertion of a continuous function, Proc. Amer. Math. Soc. 49 (1975) 90-94.

[35] E. P. Lane, Insertion of a continuous function, Topology Proc. 4 (1979) 463-478.

[36] E. P. Lane, Lebesgue sets and insertion of a continuous function, Proc. Amer. Math. Soc. 87 (1983) $539-542$.

[37] J. Mack, Countable paracompactness and weak normality properties, Trans. Amer. Math. Soc. 148 (1970) $265-272$

[38] J. Paseka and P. Sekanina, A note on extremally disconnected frames, Acta Univ. Carolin. Math. Phys. 31 (1990) $75-84$.

[39] J. Picado and A. Pultr, Locales treated mostly in a covariant way, Textos de Matemática, Vol. 41, Universidade de Coimbra, 2008.

[40] J. Picado and A. Pultr, Frames and locales: topology without points, Frontiers in Mathematics, Vol. 28, Springer, Basel, 2012

[41] G. L. Seever, Measures on F-spaces, Trans. Amer. Math. Soc. 133 (1968) 267-280.

[42] M. Singal and A. Singal, Almost normal and almost completely regular spaces, Kyungpook Math. J. 25 (1970) $141-152$.

[43] M. Singal and A. Singal, Mildly normal spaces, Kyungpook Math. J. 13 (1973) 27-31.

[44] M. H. Stone, Boundedness properties in function lattices, Canad. J. Math. 1 (1949) 176-186.

[45] H. Tietze, Über Funktionen, die auf einer abgeschlossenen menge stetig sind, J. Reine Angew. Math. 145 (1915) 9-14.

[46] H. Tong, Some characterizations of normal and perfectly normal spaces, Duke Math. J. 19 (1952) $289-292$.

[47] P. S. Urysohn, Über die Mächtigkeit der zusammen hängenden Mengen, Math. Ann. 94 (1925) 262-295.

[48] A. C. Zaanen, Riesz Spaces II, North-Holland, Amsterdam, 1983. 